\title{
A Letter of Intent to Build a MiniBooNE Near Detector:BooNE
}

\author{
October 12, 2009 \\ I. Stancu \\ University of Alabama, Tuscaloosa, AL 35487 \\ Z. Djurcic \\ Argonne National Laboratory, Argonne, IL 60439 \\ D. Smith \\ Embry-Riddle Aeronautical University, Prescott, AZ 86301 \\ R. Ford, T. Kobilarcik, W. Marsh, \& C. D. Moore \\ Fermi National Accelerator Laboratory, Batavia, IL 60510 \\ J. Grange, B. Osmanov, \& H. Ray \\ University of Florida, Gainesville, FL 32611
}
G. T. Garvey, J. A. Green, W. C. Louis, C. Mauger, G. B. Mills, Z. Pavlovic,
R. Van de Water, D. H. White, \& G. P. Zeller
Los Alamos National Laboratory, Los Alamos, NM 87545

W. Metcalf

Louisiana State University, Baton Rouge, LA 70803

B. P. Roe

University of Michigan, Ann Arbor, MI 48109

A. A. Aguilar-Arevalo

Instituto de Ciencias Nucleares, Universidad Nacional Autnoma de México, México D.F. México

\section{Executive Summary}

There is accumulating evidence for a difference between neutrino and antineutrino oscillations at the $\sim 1 \mathrm{eV}^{2}$ scale. The MiniBooNE experiment observes an unexplained excess of electron-like events at low energies in neutrino mode $\frac{1}{2}$, which may be due, for example, to either a neutral current radiative interaction 2), sterile neutrino decay $\left.{ }^{3}\right)$, or to neutrino oscillations involving sterile neutrinos $4|5| 6|7| 8|9|$ and which may be related to the LSND signal 10 . No excess of electron-like events $(-0.5 \pm 7.8 \pm 8.7)$, however, is observed so far at low energies in antineutrino mode 11). Furthermore, global $3+1$ and $3+2$ sterile neutrino fits to the world neutrino and antineutrino data ${ }^{12}$ suggest a difference between neutrinos and antineutrinos with 
significant $\left(\sin ^{2} 2 \theta_{\mu \mu} \sim 35 \%\right) \bar{\nu}_{\mu}$ disappearance. In order to test whether the lowenergy excess is due to neutrino oscillations and whether there is a difference between $\nu_{\mu}$ and $\bar{\nu}_{\mu}$ disappearance, we propose building a second MiniBooNE detector at (or moving the existing MiniBooNE detector to) a distance of $\sim 200 \mathrm{~m}$ from the Booster Neutrino Beam (BNB) production target. With identical detectors at different distances, most of the systematic errors will cancel when taking a ratio of events in the two detectors, as the neutrino flux varies as $1 / r^{2}$ to a calculable approximation. This will allow sensitive tests of oscillations for both $\nu_{e}$ and $\bar{\nu}_{e}$ appearance and $\nu_{\mu}$ and $\bar{\nu}_{\mu}$ disappearance. Furthermore, a comparison between oscillations in neutrino mode and antineutrino mode will allow a sensitive search for CP and CPT violation in the lepton sector at short baseline $\left(\Delta m^{2}>0.1 \mathrm{eV}^{2}\right)$. Finally, by comparing the rates for a neutral current (NC) reaction, such as $\mathrm{NC} \pi^{0}$ scattering or NC elastic scattering, a direct search for sterile neutrinos will be made. The initial amount of running time requested for the near detector will be a total of $\sim 2 \mathrm{E} 20$ POT divided between neutrino mode and antineutrino mode, which will provide statistics comparable to what has already been collected in the far detector. A thorough understanding of this short-baseline physics will be of great importance to future long-baseline oscillation experiments.

\section{Introduction}

Evidence for neutrino oscillations comes from solar-neutrino 13[14|15/16/17) and reactor-antineutrino experiments ${ }^{18}$, which have observed $\nu_{e}$ disappearance at $\Delta m^{2} \sim$ $8 \times 10^{-5} \mathrm{eV}^{2}$, and atmospheric-neutrino $19|20| 21 / 22$ ) and long-baseline acceleratorneutrino experiments 2324 , which have observed $\nu_{\mu}$ disappearance at $\Delta m^{2} \sim 3 \times 10^{-3}$ $\mathrm{eV}^{2}$. In addition, the LSND experiment 10 has presented evidence for $\bar{\nu}_{\mu} \rightarrow \bar{\nu}_{e}$ oscillations at the $\Delta m^{2} \sim 1 \mathrm{eV}^{2}$ scale. If all three phenomena are caused by neutrino oscillations, these three $\Delta m^{2}$ scales cannot be accommodated within an extension of the Standard Model with only three neutrino mass eigenstates. An explanation of all three mass scales with neutrino oscillations requires the addition of one or more sterile neutrinos $\frac{4|5| 6 / 7 / 8 / 9}{6}$ or further extensions of the Standard Model (e.g., 25) $)$.

The MiniBooNE experiment was designed to test the neutrino oscillation interpretation of the LSND signal in both neutrino and antineutrino modes. MiniBooNE has approximately the same $L / E_{\nu}$ as LSND but with an order of magnitude higher baseline and energy. Due to the higher energy and dissimilar event signature, MiniBooNE systematic errors are completely different from LSND errors. MiniBooNE's updated oscillation results in neutrino mode 1 show no significant excess of events at higher energies; however, a sizeable excess of events is observed at lower energies. Although the excess energy shape does not fit simple two-neutrino oscillations, the number of excess events agrees approximately with the LSND expectation. At present, with 3.4E20 POT in antineutrino mode, MiniBooNE observes no excess so far at lower energies, while at higher energies the data are inconclusive with respect 
to antineutrino oscillations suggested by the LSND data 10 .

A global fit to the world neutrino oscillation data has been performed in terms of $3+1$ and $3+2$ sterile neutrino models $\frac{12}{}$. Although a large incompatibility is observed when fitting all of the data, there is much less tension when fitting the world neutrino and antineutrino data separately. Figs. 1, 2, 3, and 4 show the allowed regions from global fits to the world neutrino and antineutrino data, assuming $3+1$ sterile neutrino models. The best neutrino fit occurs at $\Delta m_{41}^{2}=0.19 \mathrm{eV}^{2}$ with $\sin ^{2} 2 \theta_{\mu e}=$ $0.031, \sin ^{2} 2 \theta_{\mu \mu}=0.031$, and $\sin ^{2} 2 \theta_{e e}=0.034$, corresponding to a $\chi^{2}=90.5 / 90$ DF (probability $=47 \%$ ). The best antineutrino fit occurs at $\Delta m_{41}^{2}=0.915 \mathrm{eV}^{2}$ with $\sin ^{2} 2 \theta_{\mu e}=0.0043, \sin ^{2} 2 \theta_{\mu \mu}=0.35$, and $\sin ^{2} 2 \theta_{e e}=0.043$, corresponding to a $\chi^{2}=87.9 / 103 \mathrm{DF}$ (probability $=86 \%$ ). The antineutrino fit is dominated by the LSND data 10 ; however, Fig. 3 also shows that the global antineutrino data without LSND is consistent with the LSND signal and has a closed 90\% CL contour around the LSND best-fit point. CPT-conserving oscillation scenarios appear insufficient to explain all of the data. As stated in reference 12), "CPT-violating oscillations or effective CPT violation 26/9) may succeed in reconciling all short-baseline oscillation signatures, and should be pursued".

\section{MiniBooNE}

\subsection{Description of the Experiment}

A schematic drawing of the MiniBooNE experiment at FNAL is shown in Fig. 5, The experiment is fed by $8-\mathrm{GeV}$ kinetic energy protons from the Booster that interact in a 71-cm long Be target located at the upstream end of a magnetic focusing horn. The horn pulses with a current of $174 \mathrm{kA}$ and, depending on the polarity, either focuses $\pi^{+}$and $K^{+}$and defocuses $\pi^{-}$and $K^{-}$to form a pure neutrino beam or focuses $\pi^{-}$and $K^{-}$and defocuses $\pi^{+}$and $K^{+}$to form a somewhat pure antineutrino beam. The produced pions and kaons decay in a 50-m long pipe, and a fraction of the neutrinos and antineutrinos 27 interact in the MiniBooNE detector, which is located $541 \mathrm{~m}$ downstream of the Be target. For the MiniBooNE results presented here, a total of $6.5 \times 10^{20}$ POT were collected in neutrino mode and $3.4 \times 10^{20}$ POT were collected in antineutrino mode.

The MiniBooNE detector 28 ) consists of a $12.2-\mathrm{m}$ diameter spherical tank filled with approximately 800 tons of mineral oil $\left(\mathrm{CH}_{2}\right)$. A schematic drawing of the MiniBooNE detector is shown in Fig. 6. There are a total of 1280 8-inch detector phototubes (covering $10 \%$ of the surface area) and 240 veto phototubes. The fiducial volume has a 5 -m radius and corresponds to approximately 450 tons. Only $\sim 2 \%$ of the phototube channels failed over the course of the run. 


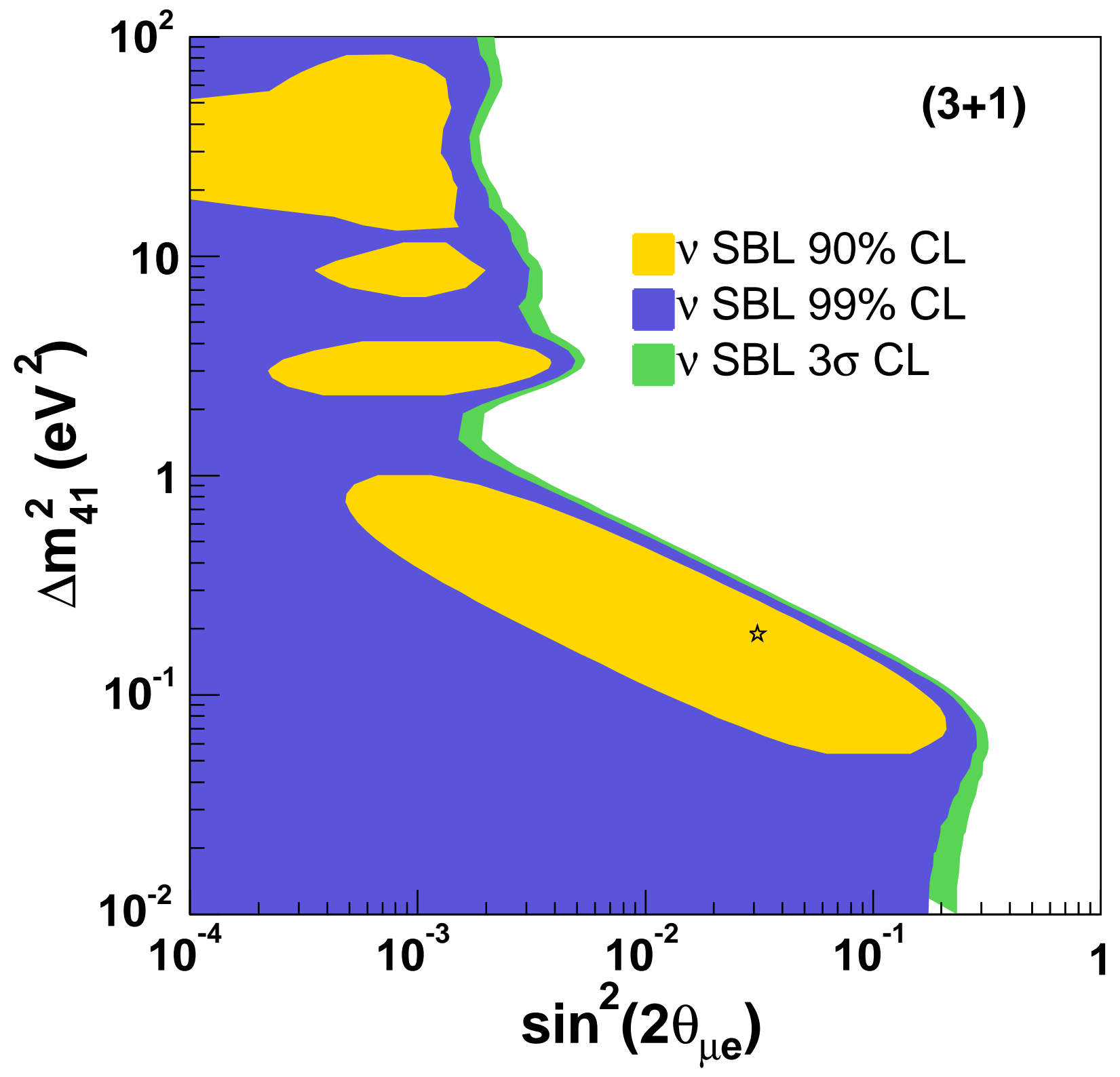

Figure 1: The allowed region for $\nu_{e}$ appearance from a global fit to the world neutrino data, assuming a $3+1$ sterile neutrino model. The star indicates the best-fit point at $\Delta m_{41}^{2}=0.19 \mathrm{eV}^{2}$ and $\sin ^{2} 2 \theta_{\mu e}=0.031$. 


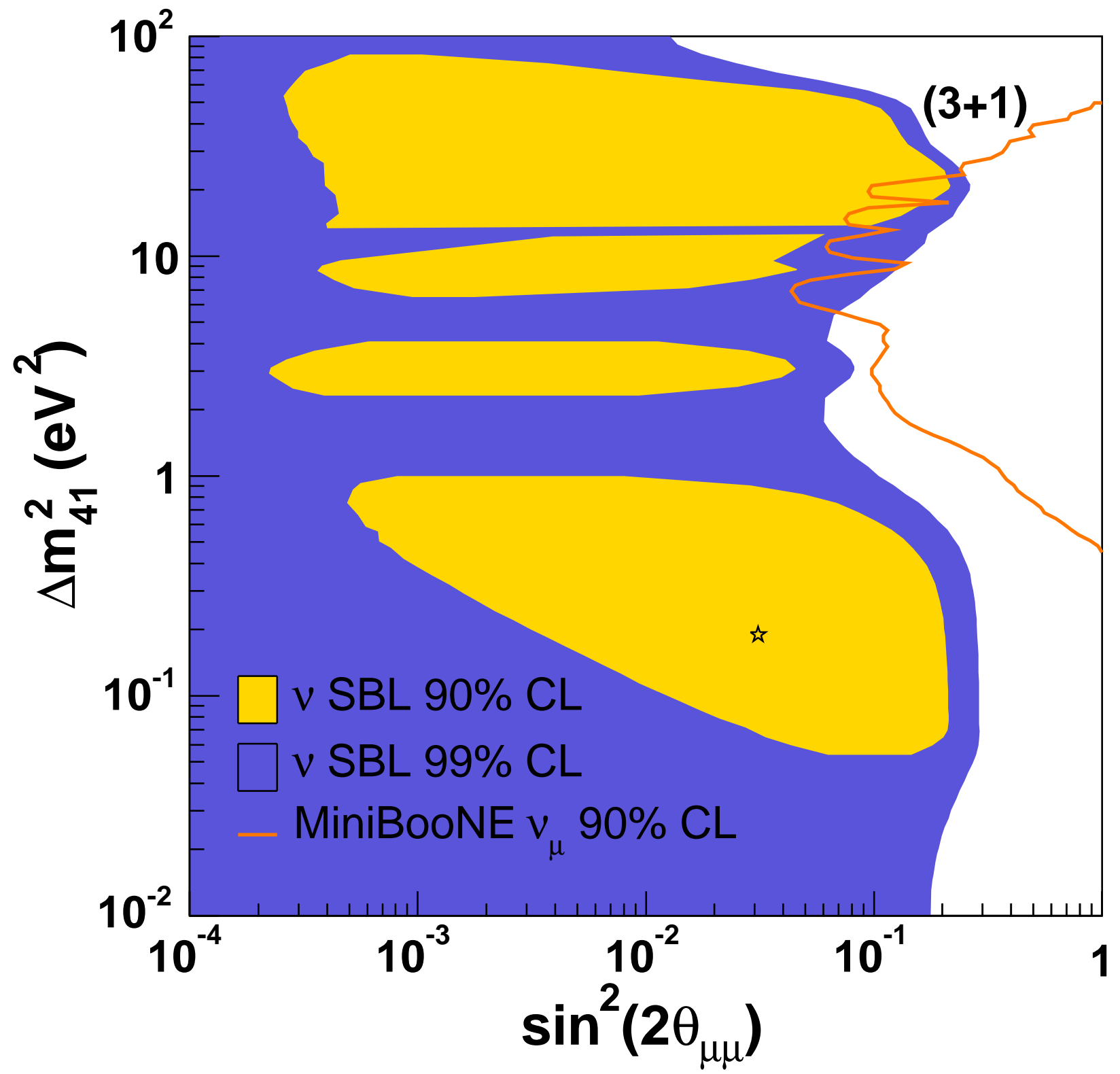

Figure 2: The allowed region for $\nu_{\mu}$ disappearance from a global fit to the world neutrino data, assuming a $3+1$ sterile neutrino model. The star indicates the best-fit point at $\Delta m_{41}^{2}=0.19 \mathrm{eV}^{2}$ and $\sin ^{2} 2 \theta_{\mu \mu}=0.031$. 


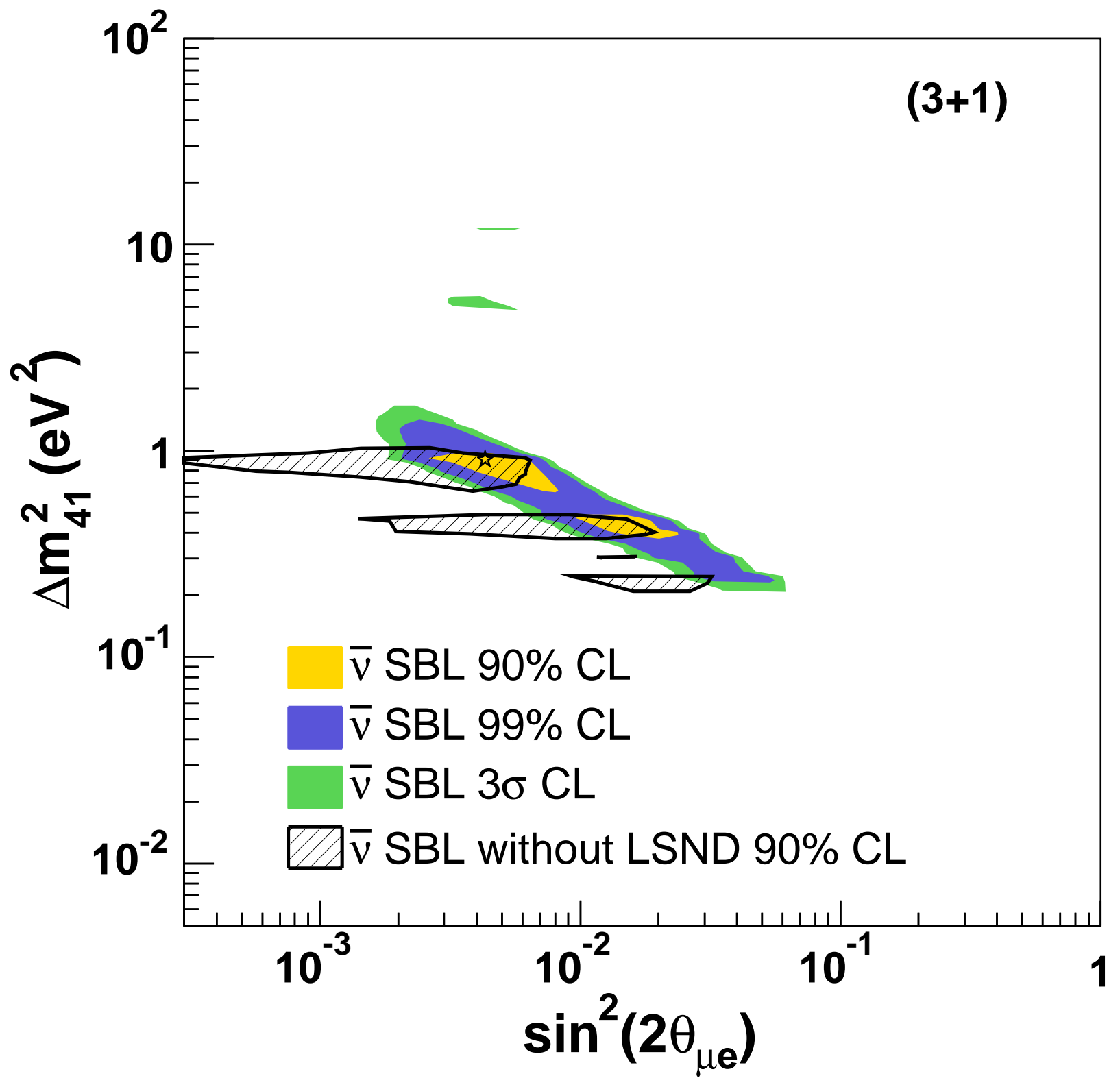

Figure 3: The allowed region for $\bar{\nu}_{e}$ appearance from a global fit to the world antineutrino data, assuming a $3+1$ sterile antineutrino model. The star indicates the best-fit point at $\Delta m_{41}^{2}=0.915$ $e V^{2}$ and $\sin ^{2} 2 \theta_{\mu e}=0.0043$. Also shown is the global fit to the world antineutrino data without LSND. 


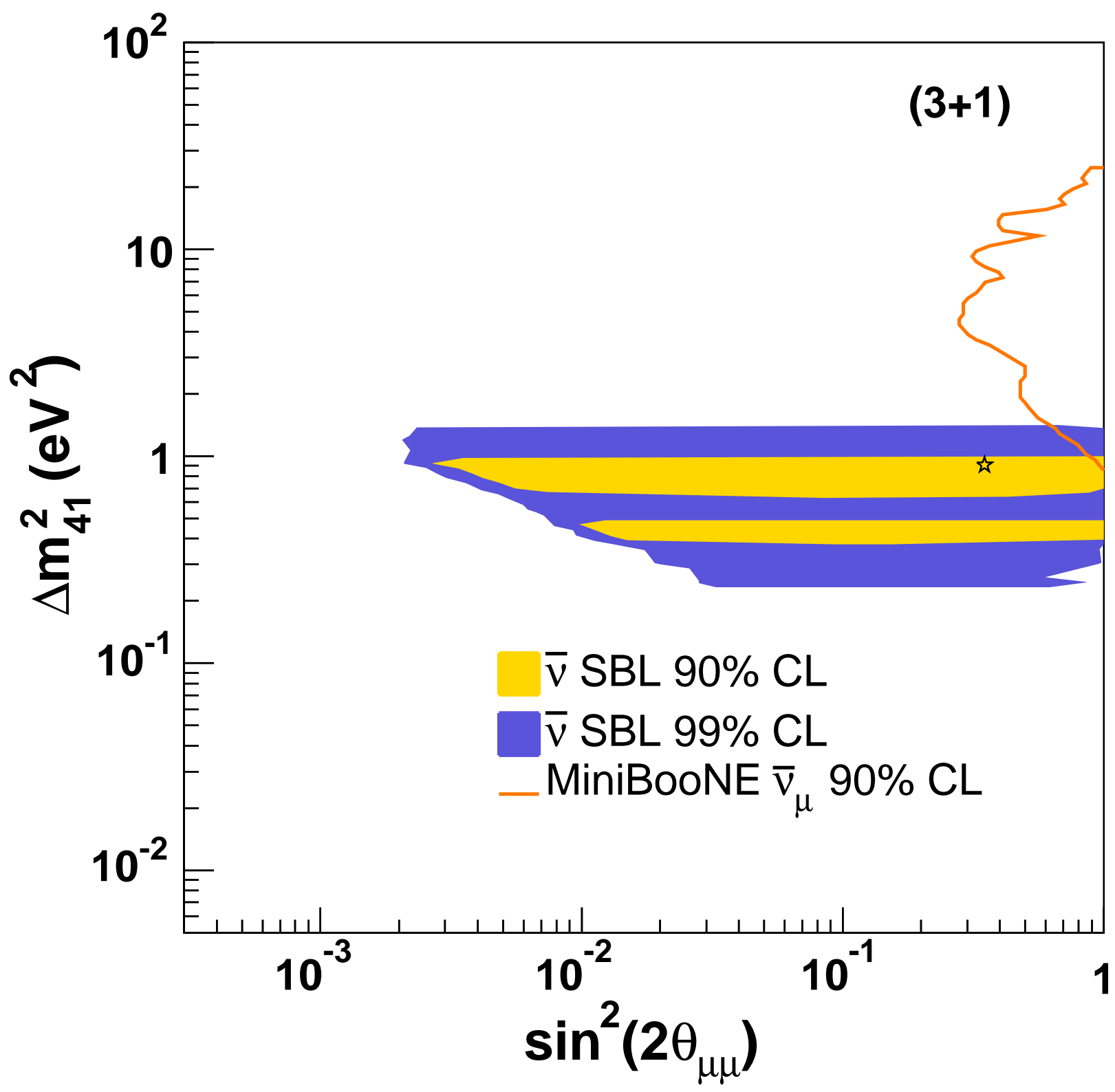

Figure 4: The allowed region for $\bar{\nu}_{\mu}$ disappearance from a global fit to the world antineutrino data, assuming a $3+1$ sterile antineutrino model. The star indicates the best-fit point at $\Delta m_{41}^{2}=0.915$ $e V^{2}$ and $\sin ^{2} 2 \theta_{\mu \mu}=0.35$. 


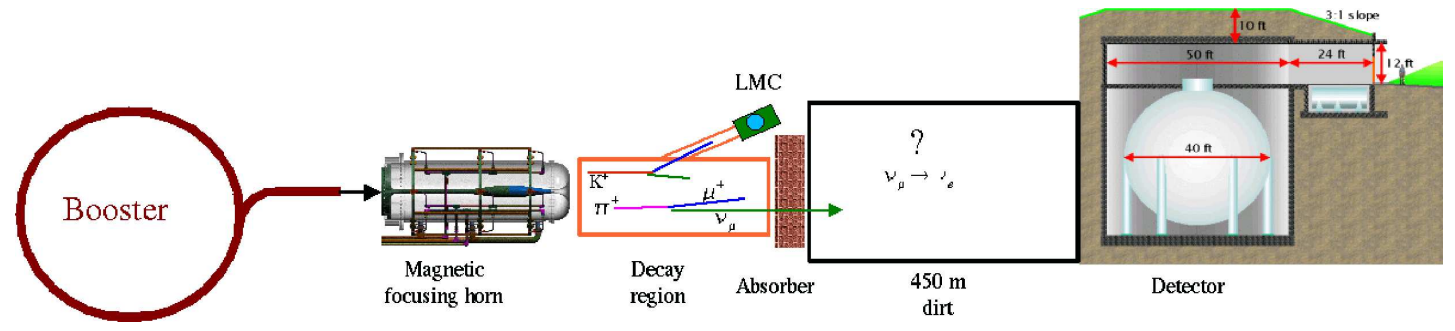

Figure 5: A schematic drawing of the MiniBooNE experiment.

\section{MiniBooNE Detector}

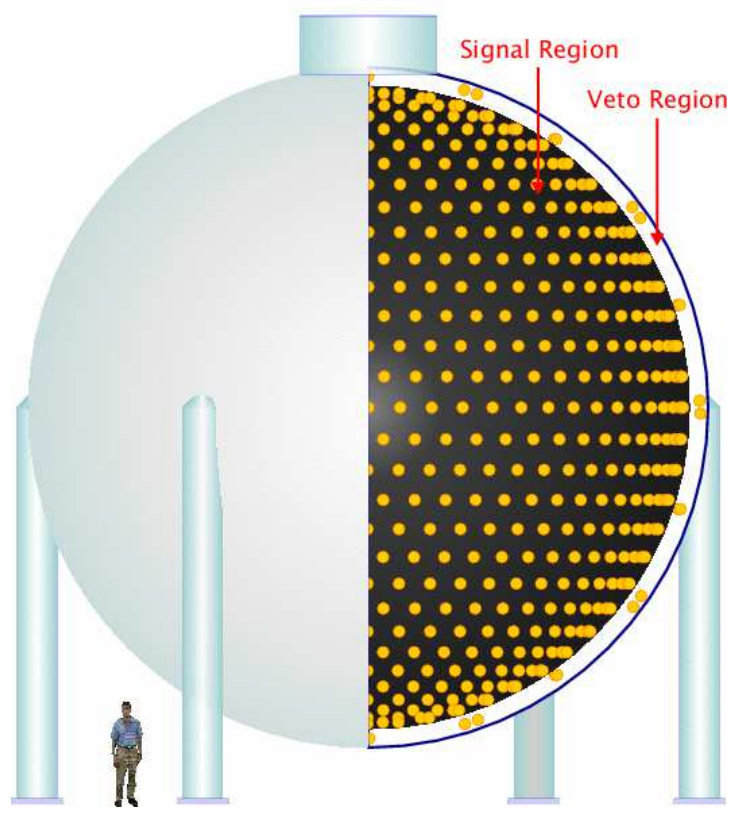

Figure 6: A schematic drawing of the MiniBooNE detector. 


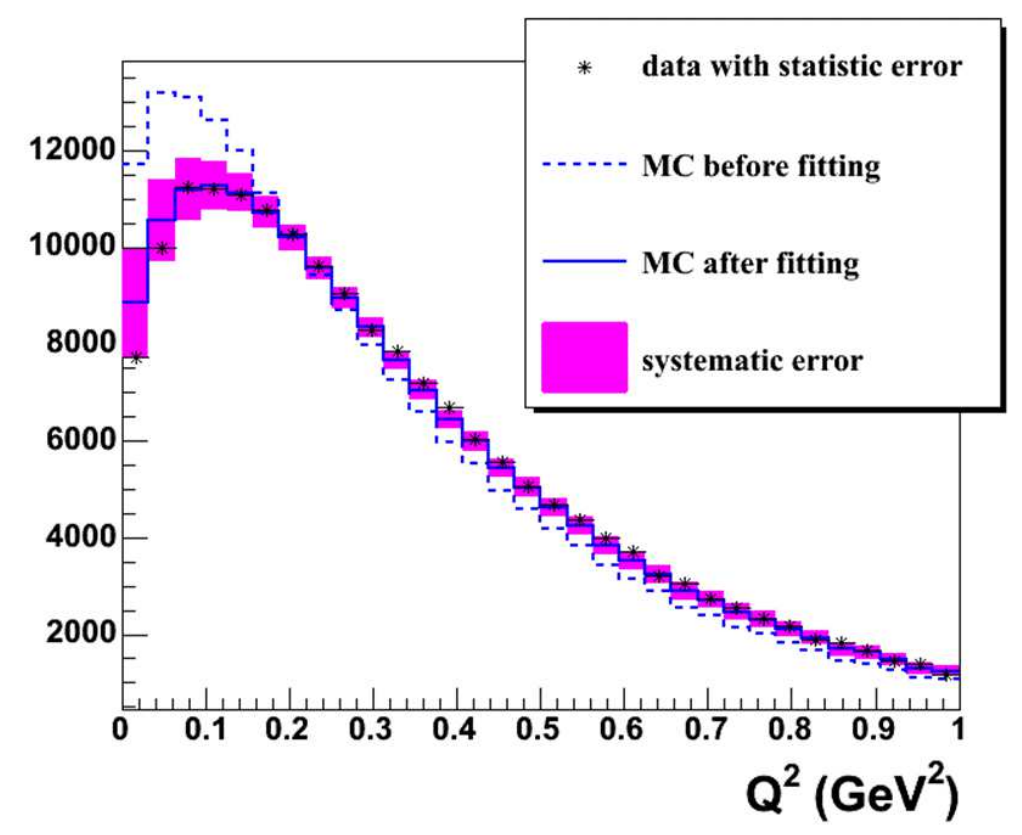

Figure 7: The $\nu_{\mu}$ CCQE $Q^{2}$ distribution for data (points with error bars) compared to the $M C$ simulation (histograms).

\subsection{MiniBooNE Cross Section Results}

MiniBooNE has published two cross section results. First, MiniBooNE has made a high-statistics measurement of $\nu_{\mu}$ charged-current quasi-elastic (CCQE) scattering events 29). Fig. 7 shows the $\nu_{\mu}$ CCQE $Q^{2}$ distribution for data (points with error bars) compared to a MC simulation (histograms). A strong disagreement between the data and the original simulation (dashed histogram) was first observed. However, by increasing the axial mass, $M_{A}$, to $1.23 \pm 0.20 \mathrm{GeV}$ and by introducing a new variable, $\kappa=1.019 \pm 0.011$, where $\kappa$ is the increase in the incident proton threshold, the agreement between data and the simulation (solid histogram) is greatly improved. It is impressive that such good agreement is obtained by adjusting these two variables.

MiniBooNE has also collected the world's largest sample of neutral-current $\pi^{0}$ events ${ }^{30}$, as shown in Fig. 8. By fitting the $\gamma \gamma$ mass and $E_{\pi}\left(1-\cos \theta_{\pi}\right)$ distributions, the fraction of $\pi^{0}$ produced coherently is determined to be $19.5 \pm 1.1 \pm 2.5 \%$. Excellent agreement is obtained between data and MC simulation.

\subsection{Neutrino Oscillation Event Selection}

MiniBooNE searches for $\nu_{\mu} \rightarrow \nu_{e}$ oscillations by measuring the rate of $\nu_{e} C \rightarrow e^{-} X$ 

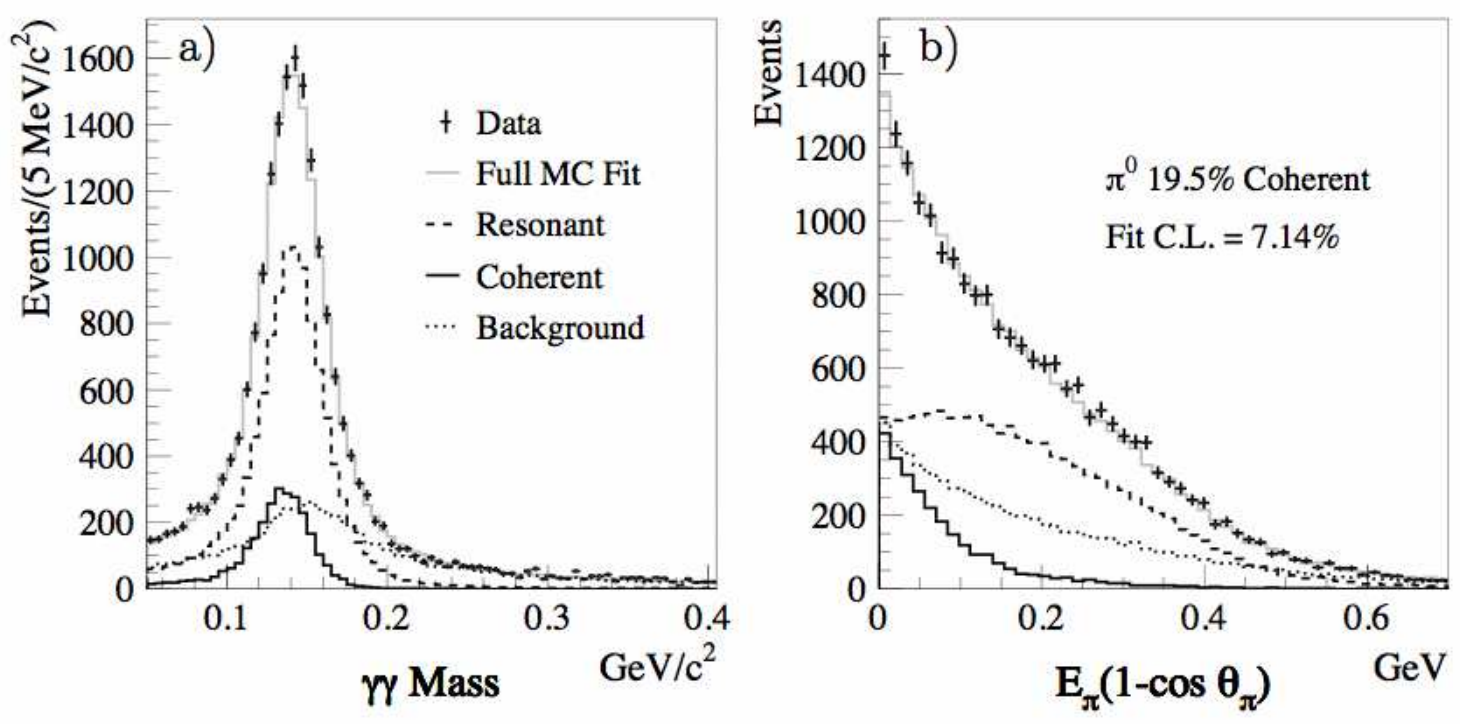

Figure 8: The neutral-current $\pi^{0} \gamma \gamma$ mass and $E_{\pi}\left(1-\cos \theta_{\pi}\right)$ distributions for data (points with error bars) compared to the MC simulation (histograms).

CCQE events and testing whether the measured rate is consistent with the estimated background rate. To select candidate $\nu_{e}$ CCQE events, an initial selection is first applied: $>200$ tank hits, $<6$ veto hits, reconstructed time within the neutrino beam spill, reconstructed vertex radius $<500 \mathrm{~cm}$, and visible energy $E_{v i s}>140$ $\mathrm{MeV}$. It is then required that the event vertex reconstructed assuming an outgoing electron and the track endpoint reconstructed assuming an outgoing muon occur at radii $<500 \mathrm{~cm}$ and $<488 \mathrm{~cm}$, respectively, to ensure good event reconstruction and efficiency for possible muon decay electrons. Particle identification (PID) cuts are then applied to reject muon and $\pi^{0}$ events. Several improvements have been made to the neutrino oscillation data analysis since the initial data was published ${ }^{1}$, including an improved background estimate, an additional fiducial volume cut that greatly reduces the background from events produced outside the tank (dirt events), and an increase in the data sample from $5.579 \times 10^{20}$ POT to $6.462 \times 10^{20}$ POT. A total of 89,200 neutrino events pass the initial selection, while 1069 events pass the complete event selection of the final analysis with $E_{\nu}^{Q E}>200 \mathrm{MeV}$, where $E_{\nu}^{Q E}$ is the reconstructed neutrino energy.

\subsection{Neutrino Oscillation Signal and Background Reactions}

Table 1 shows the expected number of candidate $\nu_{e}$ CCQE background events with $E_{\nu}^{Q E}$ between $200-300 \mathrm{MeV}, 300-475 \mathrm{MeV}$, and $475-1250 \mathrm{MeV}$ after the complete event selection of the final analysis. The background estimate includes antineutrino events, representing $<2 \%$ of the total. The total expected backgrounds for the three energy regions are $186.8 \pm 26.0$ events, $228.3 \pm 24.5$ events, and $385.9 \pm 35.7$ events, 
Table 1: The expected number of events in the $200<E_{\nu}^{Q E}<300 \mathrm{MeV}, 300<E_{\nu}^{Q E}<475 \mathrm{MeV}$, and $475<E_{\nu}^{Q E}<1250 \mathrm{MeV}$ energy ranges from all of the significant backgrounds after the complete event selection of the final analysis. Also shown are the expected number of $\nu_{e} C C Q E$ signal events for two-neutrino oscillations at the LSND best-fit solution.

\begin{tabular}{|c|c|c|c|}
\hline Process & $200-300$ & $300-475$ & $475-1250$ \\
\hline$\nu_{\mu}$ CCQE & 9.0 & 17.4 & 11.7 \\
$\nu_{\mu} e \rightarrow \nu_{\mu} e$ & 6.1 & 4.3 & 6.4 \\
$\mathrm{NC} \pi^{0}$ & 103.5 & 77.8 & 71.2 \\
$\mathrm{NC} \rightarrow N \gamma$ & 19.5 & 47.5 & 19.4 \\
Dirt Events & 11.5 & 12.3 & 11.5 \\
Other Events & 18.4 & 7.3 & 16.8 \\
\hline$\nu_{e}$ from $\mu$ Decay & 13.6 & 44.5 & 153.5 \\
$\nu_{e}$ from $K^{+}$Decay & 3.6 & 13.8 & 81.9 \\
$\nu_{e}$ from $K_{L}^{0}$ Decay & 1.6 & 3.4 & 13.5 \\
\hline Total Background & $186.8 \pm 26.0$ & $228.3 \pm 24.5$ & $385.9 \pm 35.7$ \\
\hline LSND Best-Fit Solution & $7 \pm 1$ & $37 \pm 4$ & $135 \pm 12$ \\
\hline
\end{tabular}

respectively. For $\nu_{\mu} \rightarrow \nu_{e}$ oscillations at the best-fit LSND solution of $\Delta m^{2}=1.2$ $\mathrm{eV}^{2}$ and $\sin ^{2} 2 \theta=0.003$, the expected number of $\nu_{e}$ CCQE signal events for the three energy regions are 7 events, 37 events, and 135 events, respectively.

\subsection{Updated Neutrino Oscillation Results}

Fig. 9 shows the reconstructed neutrino energy distribution for candidate $\nu_{e}$ data events (points with error bars) compared to the MC simulation (histogram) 1 , while Fig. 10 shows the event excess as a function of reconstructed neutrino energy. Good agreement between the data and the MC simulation is obtained for $E_{\nu}^{Q E}>475 \mathrm{MeV}$; however, an unexplained excess of electron-like events is observed for $E_{\nu}^{Q E}<475$ $\mathrm{MeV}$. As shown in Fig. 10, the magnitude of the excess is very similar to what is expected from neutrino oscillations based on the LSND signal. Although the shape of the excess is not consistent with simple two-neutrino oscillations, more complicated oscillation models $4 / 5 / 6 / 7819$ ) or sterile neutrino decay ${ }^{3}$ ) have shapes that may be consistent with the LSND signal. A test of the sterile neutrino decay model ${ }^{3}$ ) can be performed by searching for the decay $D_{s}^{+} \rightarrow \mu^{+} \nu_{h}$, where the heavy sterile neutrino $\nu_{h}$ has a mass around $500 \mathrm{MeV}$.

Table 2 shows the number of data, background, and excess events for different $E_{\nu}^{Q E}$ ranges, together with the excess significance. For the final analysis, an excess of $128.8 \pm 20.4 \pm 38.3$ events is observed for $200<E_{\nu}^{Q E}<475 \mathrm{MeV}$. For the entire $200<E_{\nu}^{Q E}<1250 \mathrm{MeV}$ energy region, the excess is $151.0 \pm 28.3 \pm 50.7$ events. As shown in Fig. 11, the event excess occurs for $E_{v i s}<400 \mathrm{MeV}$, where $E_{v i s}$ is the visible energy. 


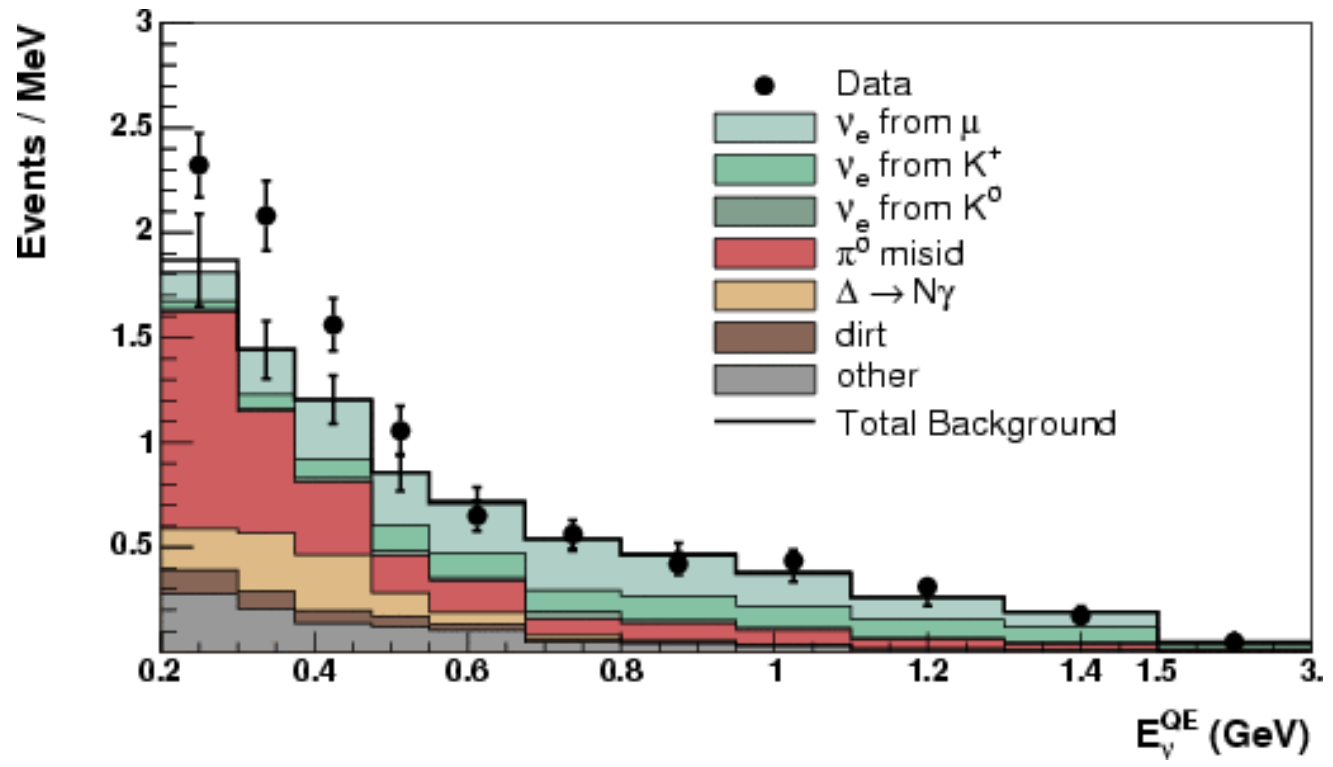

Figure 9: The MiniBooNE reconstructed neutrino energy distribution for candidate $\nu_{e}$ data events (points with error bars) compared to the Monte Carlo simulation (histogram).

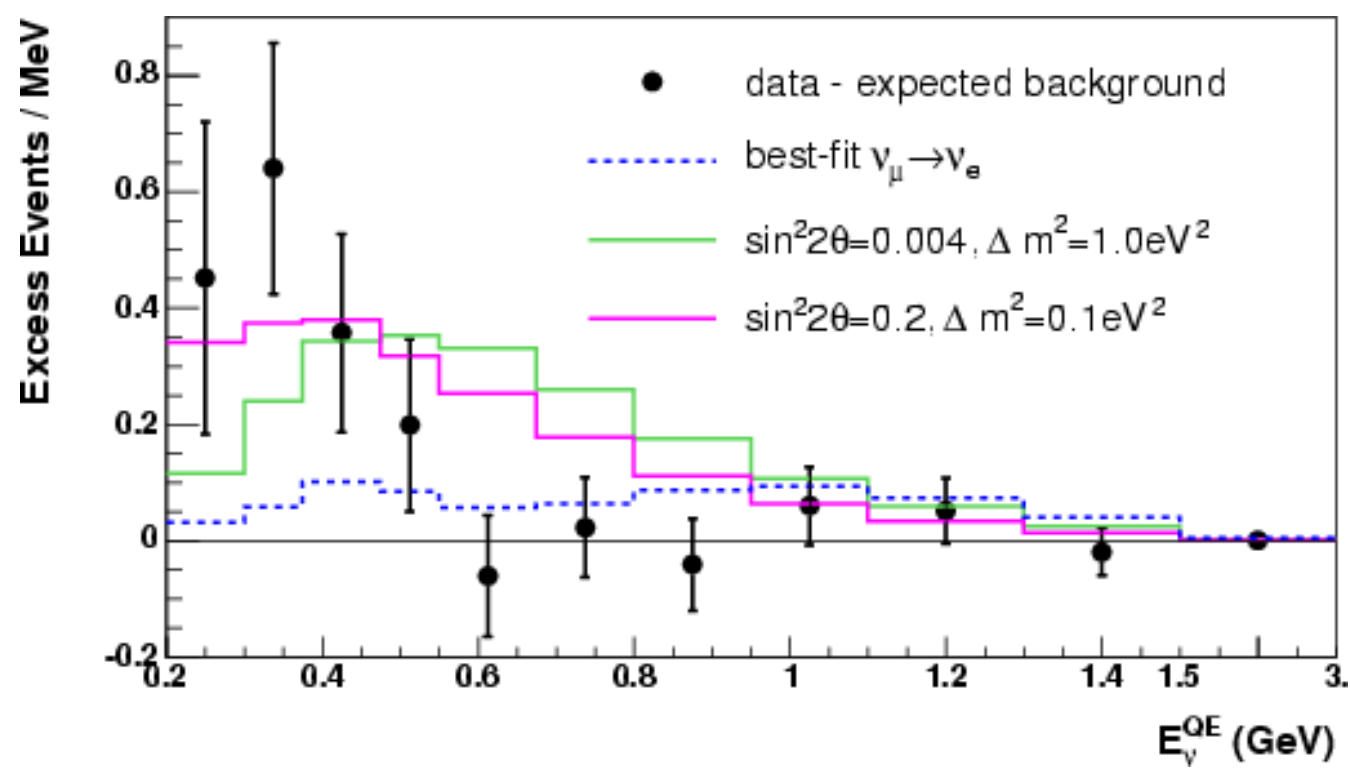

Figure 10: The event excess as a function of $E_{\nu}^{Q E}$. Also shown are the expectations from the best oscillation fit $\left(\sin ^{2} 2 \theta=0.0017, \Delta m^{2}=3.14 \mathrm{eV}^{2}\right.$ ) and from neutrino oscillation parameters in the LSND allowed region. The error bars include both statistical and systematic errors. 
Table 2: The number of data, background, and excess events for different $E_{\nu}^{Q E}$ ranges, together with the significance of the excesses in neutrino mode.

\begin{tabular}{|c|c|}
\hline Event Sample & Final Analysis \\
\hline $200-300 \mathrm{MeV}$ & 232 \\
Data & $186.8 \pm 13.7 \pm 22.1$ \\
Background & $45.2 \pm 13.7 \pm 22.1$ \\
Excess & $1.7 \sigma$ \\
Significance & 312 \\
\hline $300-475 \mathrm{MeV}$ & $3.4 \sigma$ \\
Data & 544 \\
Background & $228.3 \pm 15.1 \pm 19.3$ \\
Excess & $83.7 \pm 15.1 \pm 19.3$ \\
Significance & $20.4 \pm 38.3$ \\
\hline $200-475 \mathrm{MeV}$ & $3.0 \sigma$ \\
Data & 408 \\
Background & $415.2 \pm 38.3$ \\
Excess & $128.8 \pm 20.4 \pm 2$ \\
Significance & $0.6 \sigma$ \\
\hline $475-1250 \mathrm{MeV}$ & \\
Data & $385.9 \pm 19.6 \pm 29.8$ \\
Background & $22.1 \pm 19.6 \pm 29.8$ \\
Excess & \\
Significance & \\
\hline
\end{tabular}




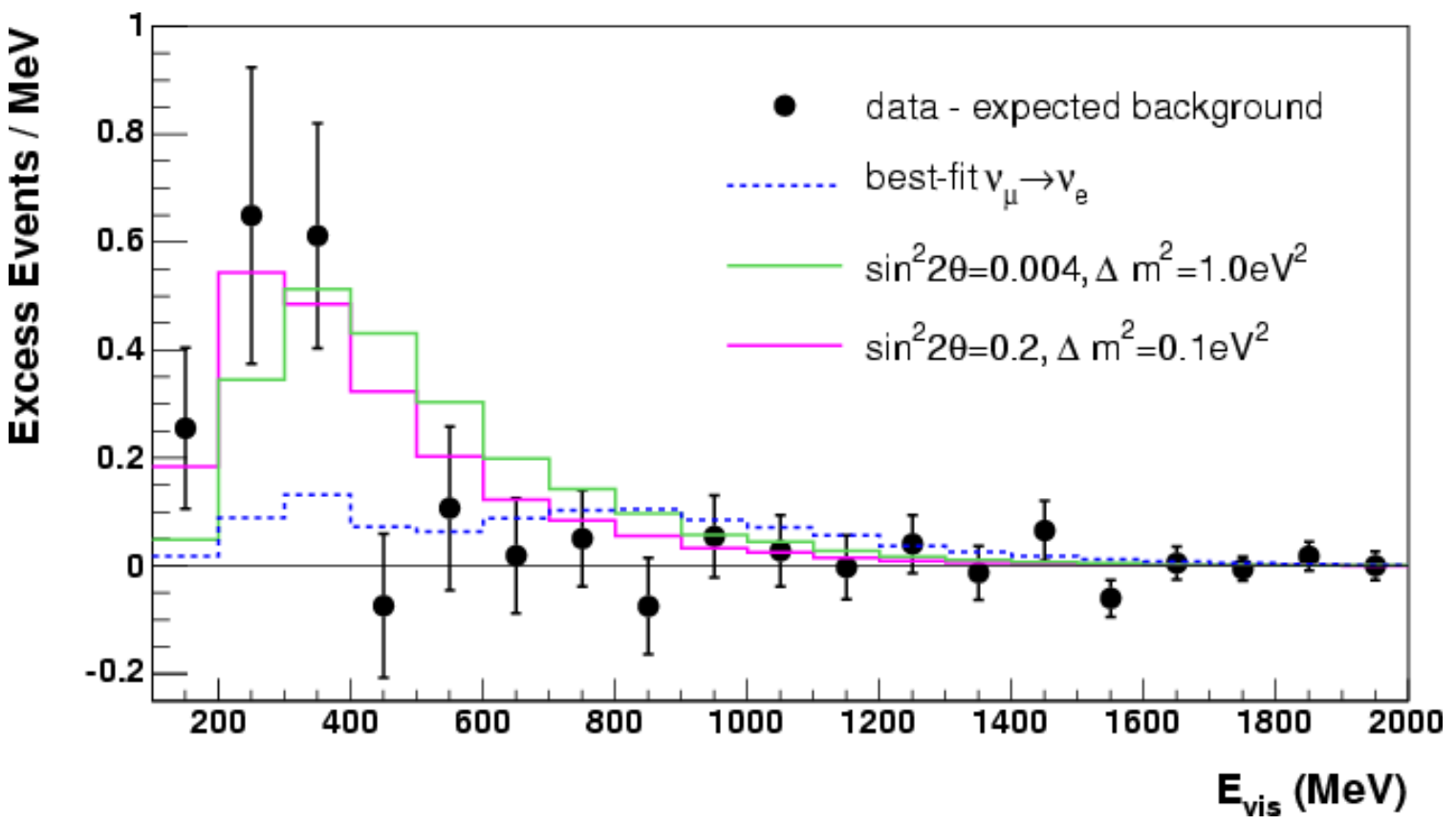

Figure 11: The event neutrino excess as a function of $E_{v i s}$ for $E_{\nu}^{Q E}>200 \mathrm{MeV}$. Also shown are the expectations from the best oscillation fit $\left(\sin ^{2} 2 \theta=0.0017, \Delta m^{2}=3.14 \mathrm{eV}^{2}\right.$ ) and from neutrino oscillation parameters in the LSND allowed region. The error bars include both statistical and systematic errors.

Figs. 12 and 13 show the event excess as functions of $Q^{2}$ and $\cos (\theta)$ for events in the $300<E_{\nu}^{Q E}<475 \mathrm{MeV}$ range, where $Q^{2}$ is determined from the energy and angle of the outgoing lepton and $\theta$ is the angle between the beam direction and the reconstructed event direction. Also shown in the figures are the expected shapes from $\nu_{e} C \rightarrow e^{-} X$ and $\bar{\nu}_{e} C \rightarrow e^{+} X$ charged-current (CC) scattering and from the NC $\pi^{0}$ and $\Delta \rightarrow N \gamma$ reactions, which are representative of photon events produced by $\mathrm{NC}$ scattering. The NC scattering assumes the $\nu_{\mu}$ energy spectrum, while the $\mathrm{CC}$ scattering assumes the transmutation of $\nu_{\mu}$ into $\nu_{e}$ and $\bar{\nu}_{e}$, respectively. As shown in Table 3, the $\chi^{2}$ values from comparisons of the event excess to the expected shapes are acceptable for all of the processes. However, any of the backgrounds in Table 3 would have to be increased by $>5 \sigma$ to explain the low-energy excess.

\subsection{Initial Antineutrino Oscillation Results}

The same analysis that was used for the neutrino oscillation results is employed for the initial antineutrino oscillation results 11). Fig. 14 shows the estimated neutrino fluxes for neutrino mode and antineutrino mode, respectively. The fluxes are fairly similar (the intrinsic electron-neutrino background is approximately $0.5 \%$ for both modes of running), although the wrong-sign contribution to the flux in antineutrino mode $(\sim 18 \%)$ is much larger than in neutrino mode $(\sim 6 \%)$. The average $\nu_{e}$ plus $\bar{\nu}_{e}$ 


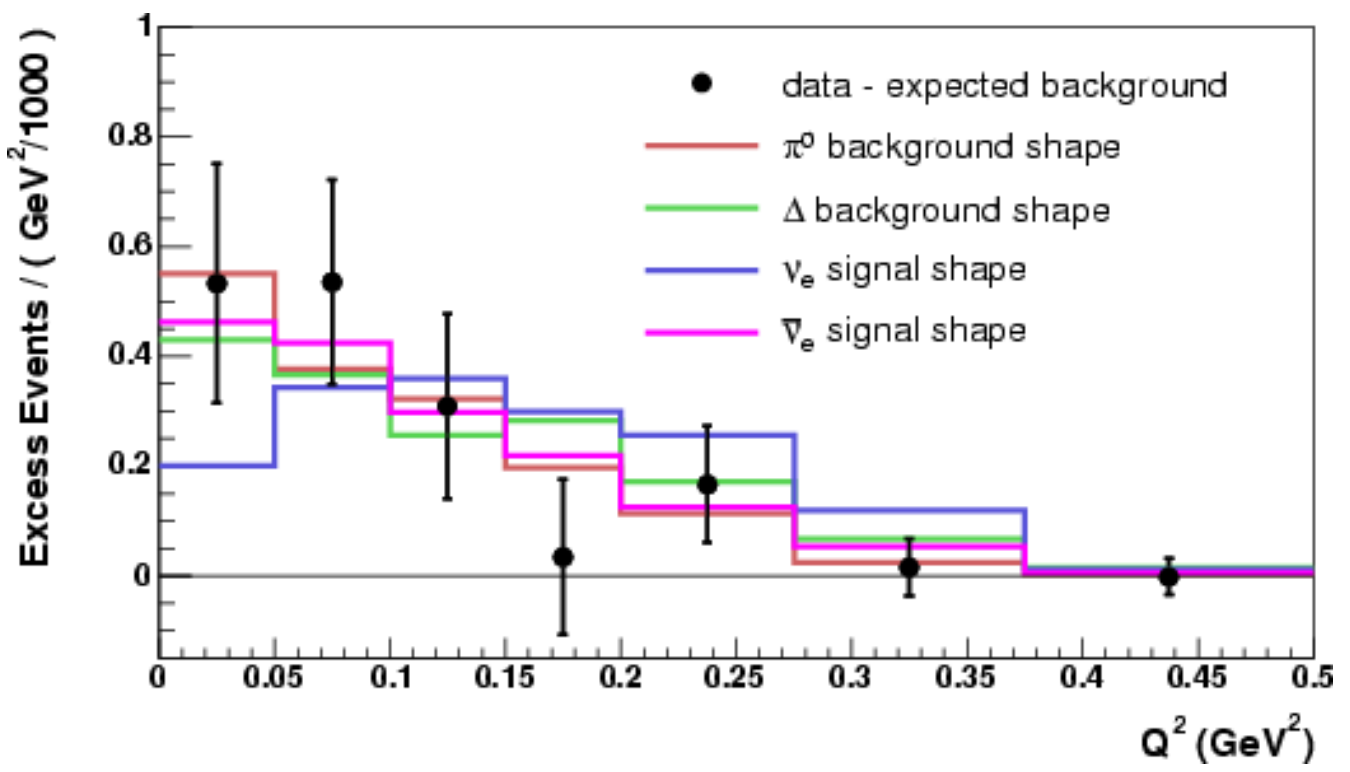

Figure 12: The neutrino event excess as a function of $Q^{2}$ for $300<E_{\nu}^{Q E}<475 \mathrm{MeV}$.

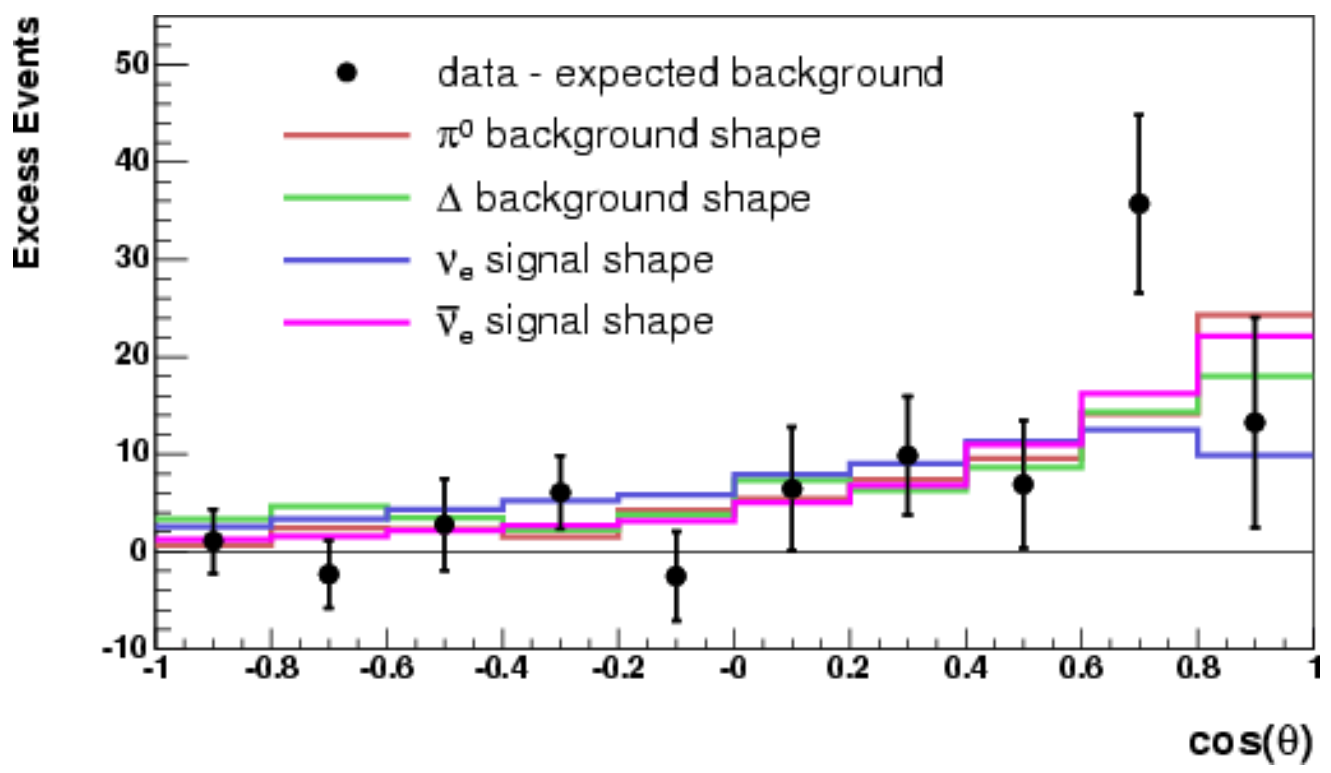

Figure 13: The neutrino event excess as a function of $\cos (\theta)$ for $300<E_{\nu}^{Q E}<475 \mathrm{MeV}$. 
Table 3: The $\chi^{2}$ values from comparisons of the neutrino event excess $Q^{2}$ and $\cos (\theta)$ distributions for $300<E_{\nu}^{Q E}<475 \mathrm{MeV}$ to the expected shapes from various $N C$ and $C C$ reactions. Also shown is the factor increase necessary for the estimated background for each process to explain the low-energy excess and the corresponding number of sigma.

\begin{tabular}{|c|c|c|c|}
\hline Process & $\chi^{2}(\cos \theta) / 9 \mathrm{DF}$ & $\chi^{2}\left(Q^{2}\right) / 6 \mathrm{DF}$ & Factor Increase \\
\hline $\mathrm{NC} \pi^{0}$ & 13.46 & 2.18 & $2.0(6.8 \sigma)$ \\
$\Delta \rightarrow N \gamma$ & 16.85 & 4.46 & $2.7(18.4 \sigma)$ \\
$\nu_{e} C \rightarrow e^{-} X$ & 14.58 & 8.72 & $2.4(15.3 \sigma)$ \\
$\bar{\nu}_{e} C \rightarrow e^{+} X$ & 10.11 & 2.44 & $65.4(41.0 \sigma)$ \\
\hline
\end{tabular}

energies are $0.96 \mathrm{GeV}$ in neutrino mode and $0.77 \mathrm{GeV}$ in antineutrino mode, while the average $\nu_{\mu}$ plus $\bar{\nu}_{\mu}$ energies are $0.79 \mathrm{GeV}$ in neutrino mode and $0.66 \mathrm{GeV}$ in antineutrino mode. Also, as shown in Fig. 15, the estimated backgrounds in the two modes are very similar, especially at low energy. Fig. 16 shows the expected antineutrino oscillation sensitivity for the present data sample corresponding to 3.4E20 POT. The two sensitivity curves correspond to threshold neutrino energies of $200 \mathrm{MeV}$ and 475 $\mathrm{MeV}$.

The initial oscillation results for antineutrino mode are shown in Table 4 and Figs. 17 through 19. It is remarkable that no excess $(-0.5 \pm 7.8 \pm 8.7$ events $)$ is observed in the low-energy range $200<E_{\nu}^{Q E}<475 \mathrm{MeV}$. In order to understand the implications that the antineutrino data have on the neutrino low-energy excess, Table 5 shows the expected excess of low-energy events in antineutrino mode under various hypotheses. These hypotheses include the following:

- Same $\sigma$ : Same cross section for neutrinos and antineutrinos.

- $\pi^{0}$ Scaled: Scaled to number of neutral-current $\pi^{0}$ events.

- POT Scaled: Scaled to number of POT.

- BKGD Scaled: Scaled to total background events.

- CC Scaled: Scaled to number of charged-current events.

- Kaon Scaled: Scaled to number of low-energy kaon events.

- Neutrino Scaled: Scaled to number of neutrino events.

Also shown in the Table is the probability (from a two-parameter fit to the data) that each hypothesis explains the observed number of low-energy neutrino and antineutrino events, assuming only statistical errors, correlated systematic errors, and uncorrelated systematic errors. A proper treatment of the systematic errors is in progress; however, it is clear from the Table that the "Neutrino Scaled" hypothesis fits best and that the "Same $\sigma$ ", "POT Scaled", and "Kaon Scaled" hypotheses 

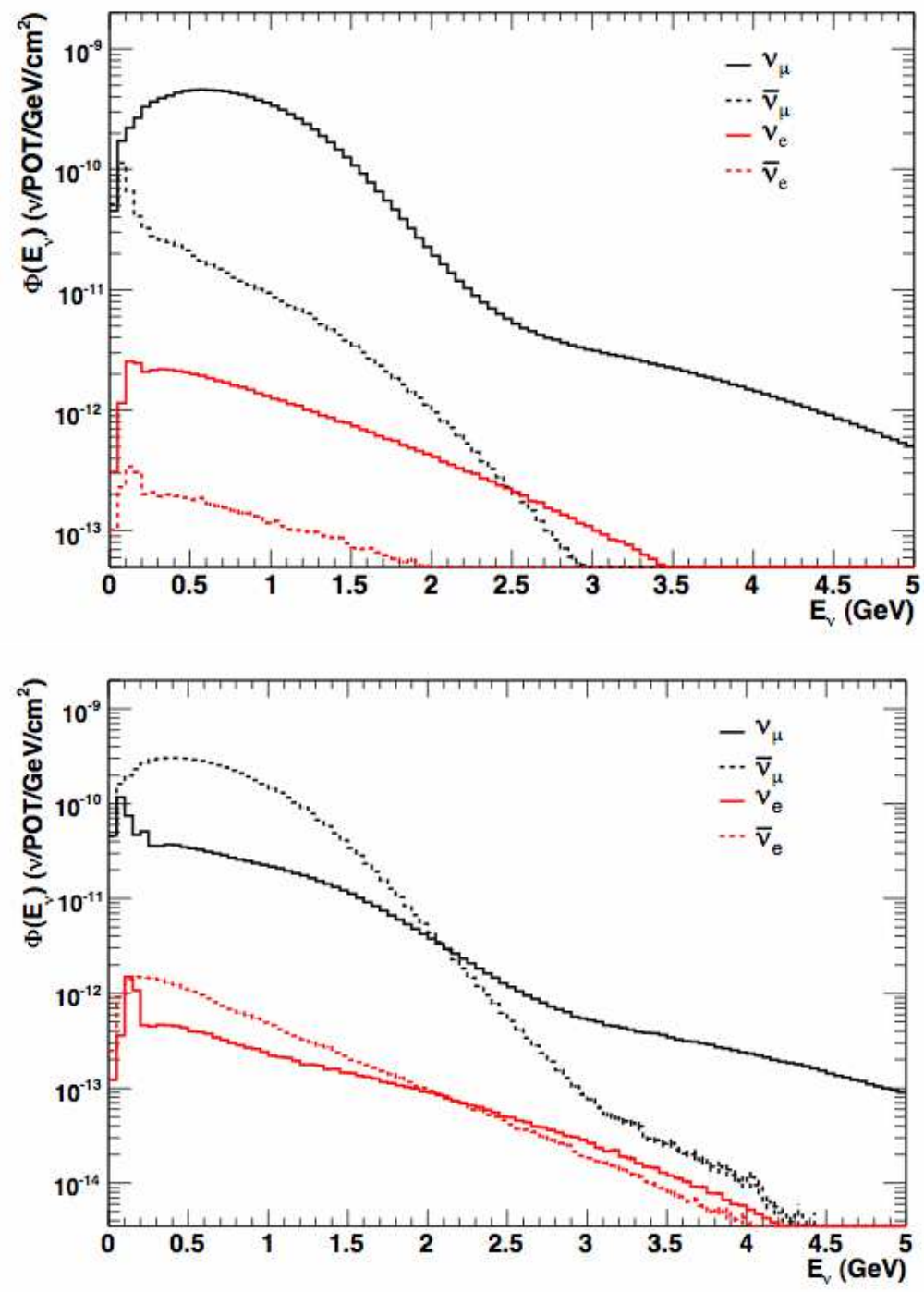

Figure 14: The estimated neutrino fluxes for neutrino mode (top plot) and antineutrino mode (bottom plot). 

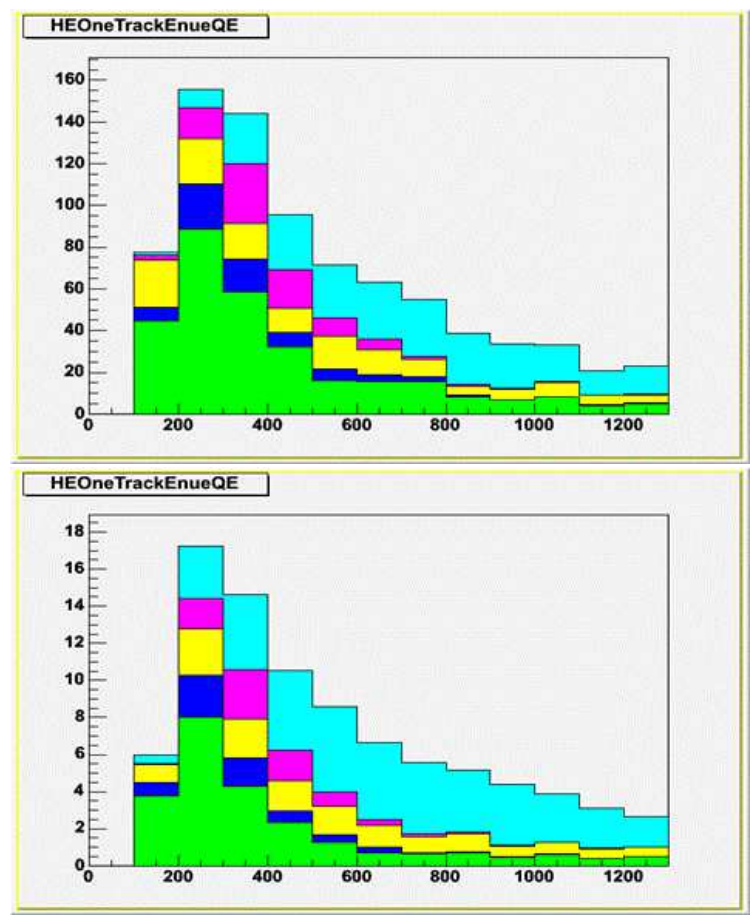

Figure 15: The estimated backgrounds for the neutrino oscillation search in neutrino mode (top plot) and antineutrino mode (bottom plot). The $\pi^{0}, \Delta \rightarrow N \gamma$, intrinsic $\nu_{e} / \bar{\nu}_{e}$, external event, and other backgrounds correspond to the green, pink, light blue, blue, and yellow colors, respectively.

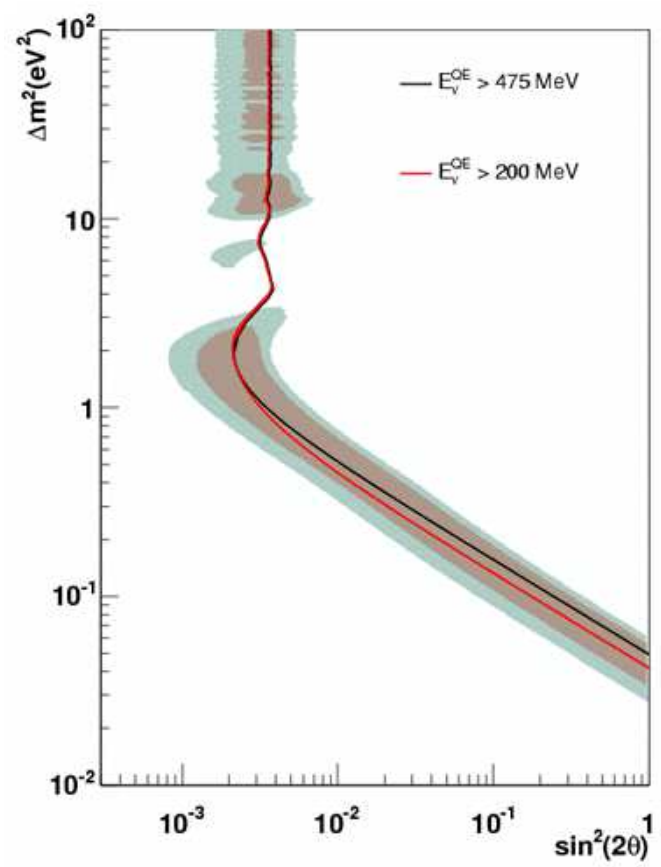

Figure 16: The expected antineutrino oscillation sensitivity at 90\% CL for the present data sample corresponding to 3.4E20 POT. The two sensitivity curves correspond to threshold energies of 200 $\mathrm{MeV}$ (red curve) and $475 \mathrm{MeV}$ (black curve). 
Table 4: The number of antineutrino data, background, and excess events for different $E_{\bar{\nu}}^{Q E}$ ranges, together with the significance of the excesses in antineutrino mode.

\begin{tabular}{|c|c|}
\hline Event Sample & Final Analysis \\
\hline $200-475 \mathrm{MeV}$ & \\
Data & 61 \\
Background & $61.5 \pm 7.8 \pm 8.7$ \\
Excess & $-0.5 \pm 7.8 \pm 8.7$ \\
Significance & $-0.04 \sigma$ \\
\hline $475-1250 \mathrm{MeV}$ & \\
Data & 61 \\
Background & $57.8 \pm 7.6 \pm 6.5$ \\
Excess & $3.2 \pm 7.6 \pm 6.5$ \\
Significance & $0.3 \sigma$ \\
\hline $475-3000 \mathrm{MeV}$ & \\
Data & 83 \\
Background & $77.4 \pm 8.8 \pm 9.6$ \\
Excess & $5.6 \pm 8.8 \pm 9.6$ \\
Significance & $0.4 \sigma$ \\
\hline
\end{tabular}

are strongly disfavored. It will be very important to understand this unexpected difference between neutrino and antineutrino properties.

The antineutrino data were also fit for oscillations in the energy range $475<$ $E_{\bar{\nu}}^{Q E}<3000 \mathrm{MeV}$, assuming antineutrino oscillations but no neutrino oscillations. The antineutrino oscillation allowed region is shown in Fig. 20, At present, the oscillation limit is worse than the sensitivity. The best oscillation fit corresponds to $\Delta m^{2}=4.4 \mathrm{eV}^{2}, \sin ^{2} 2 \theta=0.0047$, and a fitted excess of $18.6 \pm 13.2$ events, which is consistent with the LSND best-fit point of $\Delta m^{2}=1.2 \mathrm{eV}^{2}, \sin ^{2} 2 \theta=0.003$, and an expected excess of 14.7 events. With the present antineutrino statistics, the data are consistent with both the LSND best-fit point and the null point, although the LSND best-fit point has a better $\chi^{2}\left(\chi^{2}=17.63 / 15 \mathrm{DF}\right.$, probability $\left.=30 \%\right)$ than the null point $\left(\chi^{2}=22.19 / 15 \mathrm{DF}\right.$, probability $\left.=10 \%\right)$.

\subsection{MiniBooNE NuMI Results}

Neutrino events are also observed in MiniBooNE from the NuMI beam 31 . The NuMI beam, as shown in Fig. 21, differs from the Booster neutrino beam (BNB) in several respects. First, the NuMI beam is off axis by $110 \mathrm{mrad}$, whereas the BNB is on axis. Second, neutrinos from NuMI travel $\sim 700 \mathrm{~m}$, compared to $\sim 500 \mathrm{~m}$ for neutrinos from the BNB. Also, the NuMI beam has a $6 \%$ contribution from electronneutrinos and a $14 \%$ contribution from antineutrinos, while the BNB percentages are $0.5 \%$ and $2 \%$, respectively. Fig. 22 shows the estimated neutrino flux at the 


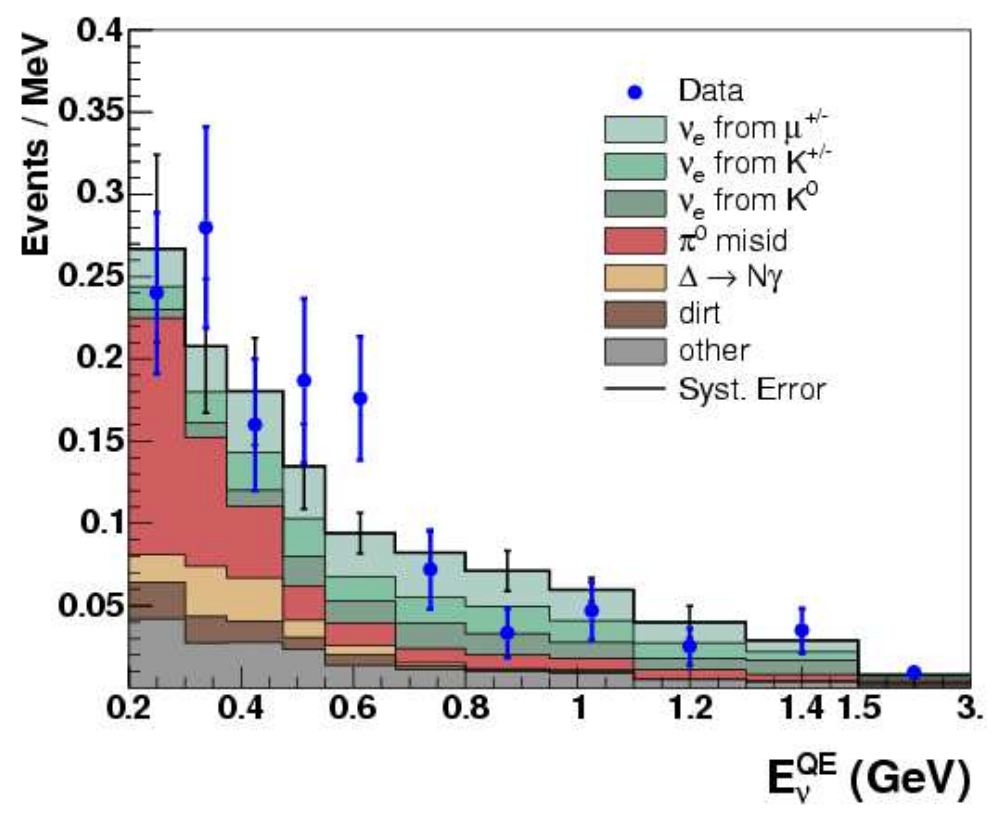

Figure 17: The MiniBooNE reconstructed antineutrino energy distribution for candidate $\bar{\nu}_{e}$ data events (points with error bars) compared to the Monte Carlo simulation (histogram).

Table 5: The expected excess of low-energy events in antineutrino mode under various hypotheses for 3.4E20 POT. Also shown in the Table is the probability (from a two-parameter fit to the data) that each hypothesis explains the observed number of low-energy neutrino and antineutrino events, assuming only statistical errors, correlated systematic errors, and uncorrelated systematic errors.

\begin{tabular}{|c|c|c|c|c|}
\hline Hypothesis & $\#$ of $\bar{\nu}$ Events & Stat. Err. & Cor. Syst. Err. & Uncor. Syst. Err. \\
\hline Same $\sigma$ & 37.2 & $0.1 \%$ & $0.1 \%$ & $6.7 \%$ \\
$\pi^{0}$ Scaled & 19.4 & $3.6 \%$ & $6.4 \%$ & $21.5 \%$ \\
POT Scaled & 67.5 & $0.0 \%$ & $0.0 \%$ & $1.8 \%$ \\
BKGD Scaled & 20.9 & $2.7 \%$ & $4.7 \%$ & $19.2 \%$ \\
CC Scaled & 20.4 & $2.9 \%$ & $5.2 \%$ & $19.9 \%$ \\
Kaon Scaled & 39.7 & $0.1 \%$ & $0.1 \%$ & $5.9 \%$ \\
Neutrino Scaled & 6.7 & $38.4 \%$ & $51.4 \%$ & $58.0 \%$ \\
\hline
\end{tabular}



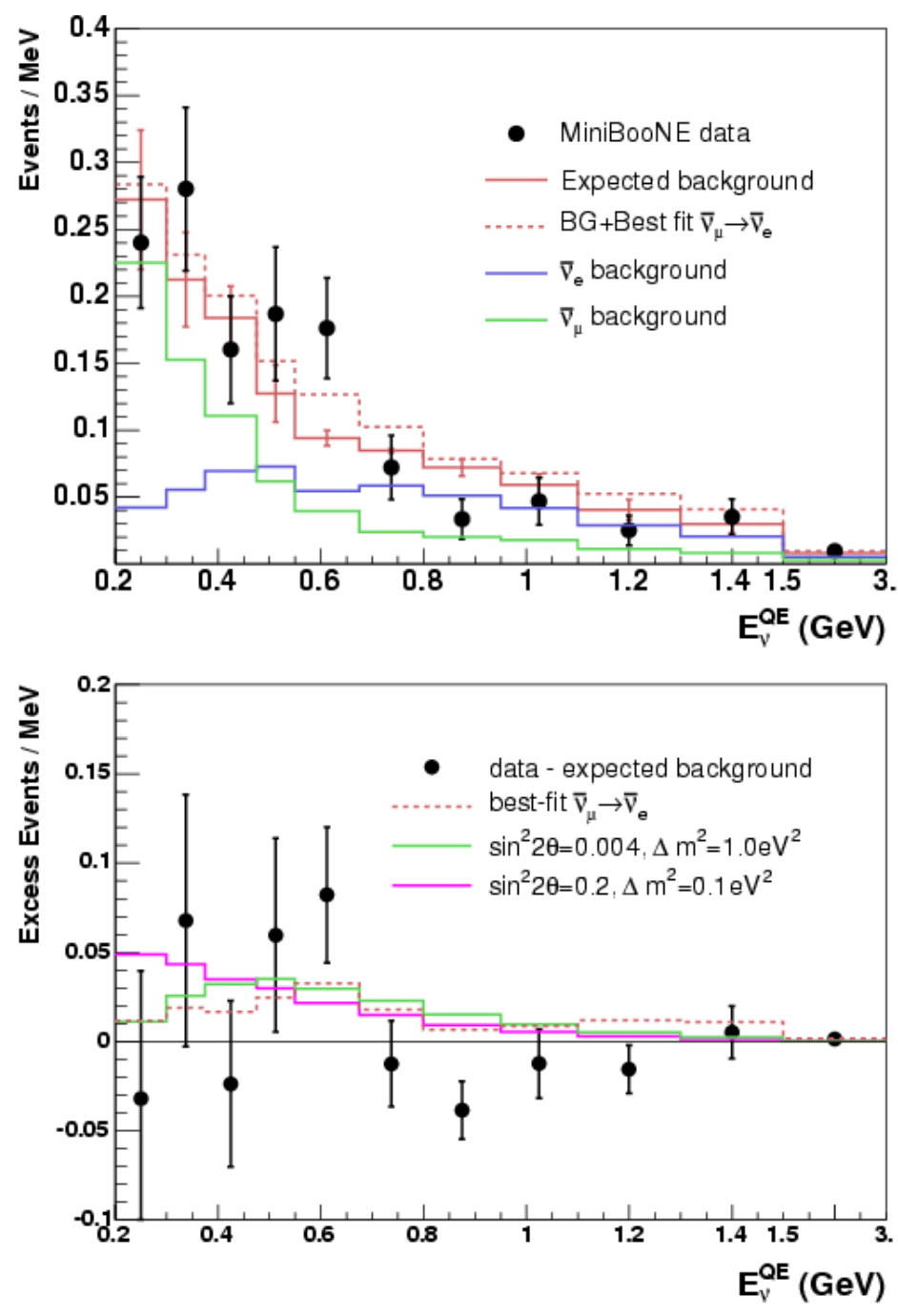

Figure 18: The MiniBooNE reconstructed antineutrino energy distribution for candidate $\bar{\nu}_{e}$ data events (top) and the excess number of events (bottom) as a function of reconstructed neutrino energy for the present antineutrino data sample corresponding to 3.4E20 POT. Also shown are the expectations from the best oscillation fit and from oscillation parameters in the LSND allowed region. 


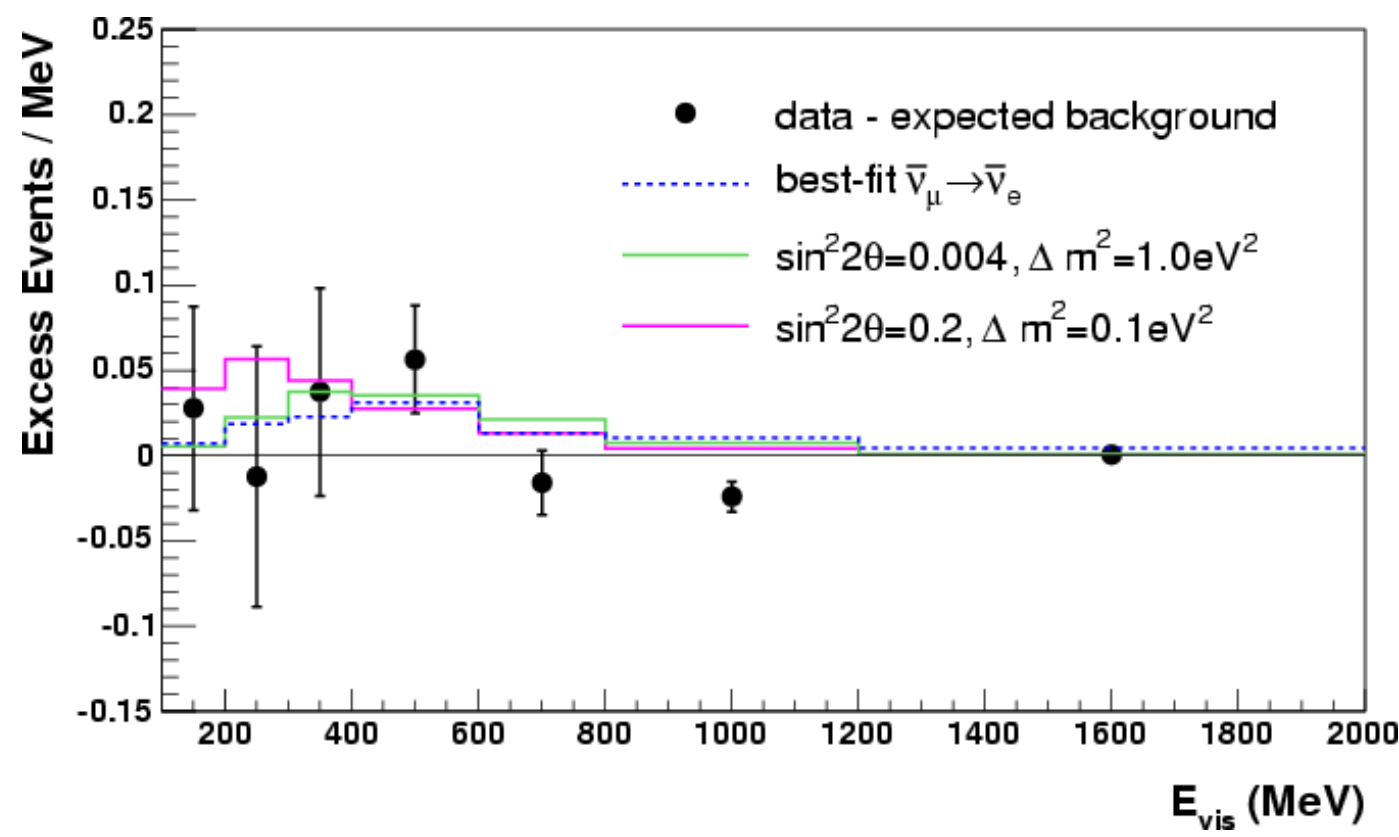

Figure 19: The excess number of candidate $\bar{\nu}_{e}$ events (data minus Monte Carlo expectation) as a function of visible energy for the present antineutrino data sample corresponding to 3.4E20 POT. Also shown are the expectations from the best oscillation fit and from oscillation parameters in the LSND allowed region.

MiniBooNE detector from the NuMI beam, while Fig. 23 compares the neutrino fluxes from the BNB and NuMI beams.

Figs. 24 and 25 show the comparison between data events (points with error bars) and the $\mathrm{MC}$ simulation (histogram) for $\nu_{\mu}$ CCQE candidate events and $\nu_{e}$ CCQE candidate events, respectively. Although the systematic errors are presently large, the data are observed to be systematically low for $\nu_{\mu}$ CCQE candidate events and systematically high for $\nu_{e}$ CCQE candidate events. Updated results should be available soon with three times the data sample and with reduced systematic errors by constraining the normalization to the $\nu_{\mu}$ sample.

The NuMI data analysis is currently directed toward examining the low-energy region and searching for neutrino oscillations. This will complement the analysis done with MiniBooNE using neutrino and anti-neutrino BNB data, but with different systematic errors. It is worth noting that the NuMI $\nu_{e}$ CCQE sample has a very different composition when compared to the BNB neutrino $\nu_{e}$ CCQE sample. The $\mathrm{BNB} \nu_{e}$ CCQE sample originates mostly from decays of pions and muons and contains a large fraction of $\nu_{\mu}$-induced mis-identified events. On other hand, the NuMI $\nu_{e}$ CCQE sample is produced mostly from the decay of kaons and contains a dominant fraction of intrinsic $\nu_{e}$ events. The analysis will be done by forming a correlation between the $\nu_{\mu} \mathrm{CCQE}$ and $\nu_{e} \mathrm{CCQE}$ samples and by tuning the prediction to the data simultaneously. The result is that common systematics cancel, and this might 


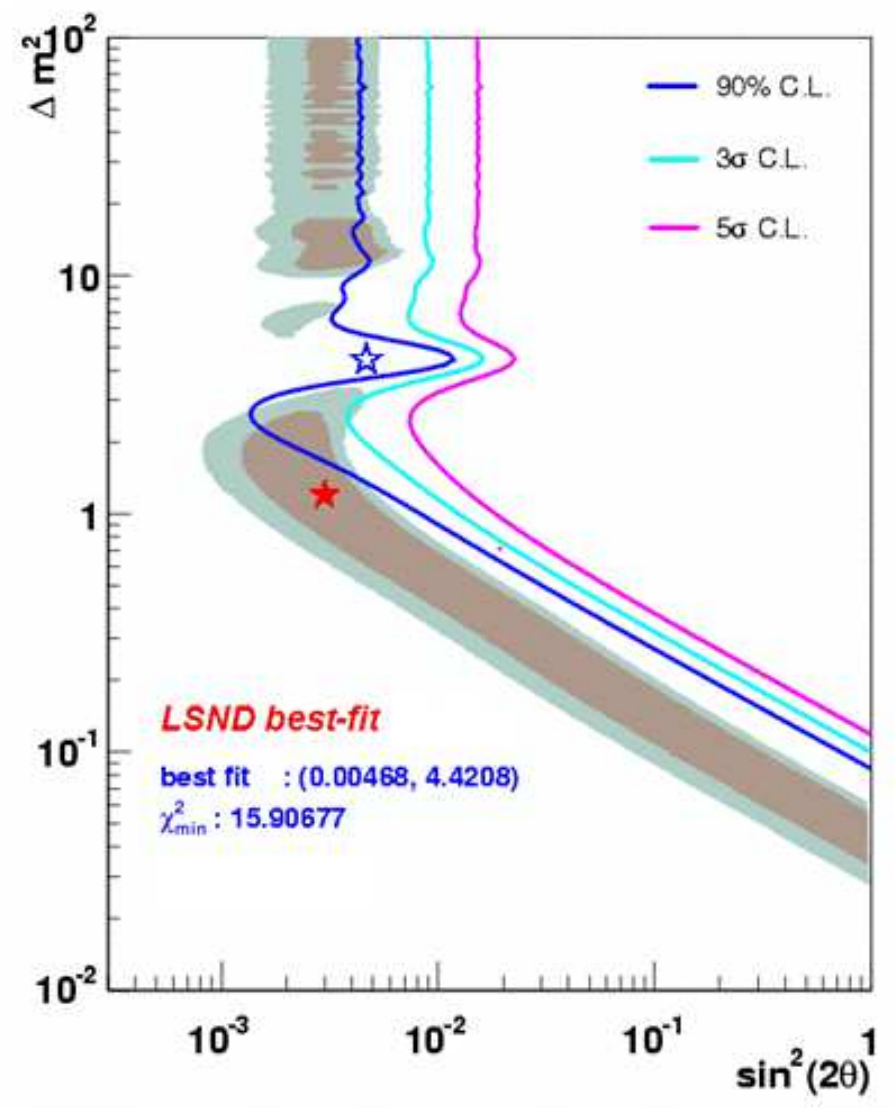

Figure 20: The antineutrino oscillation allowed region in the energy range $475<E_{\bar{\nu}}^{Q E}<3000 \mathrm{MeV}$ for the present antineutrino data sample corresponding to 3.4 E20 POT. Also shown are the best oscillation fit $\left(\Delta m^{2}=4.4 \mathrm{eV}^{2}, \sin ^{2} 2 \theta=0.0047\right.$, corresponding to an excess of $18.6 \pm 13.2$ events) and the LSND best-fit point $\left(\Delta m^{2}=1.2 e^{2}, \sin ^{2} 2 \theta=0.003\right.$, corresponding to an excess of 14.7 events). 


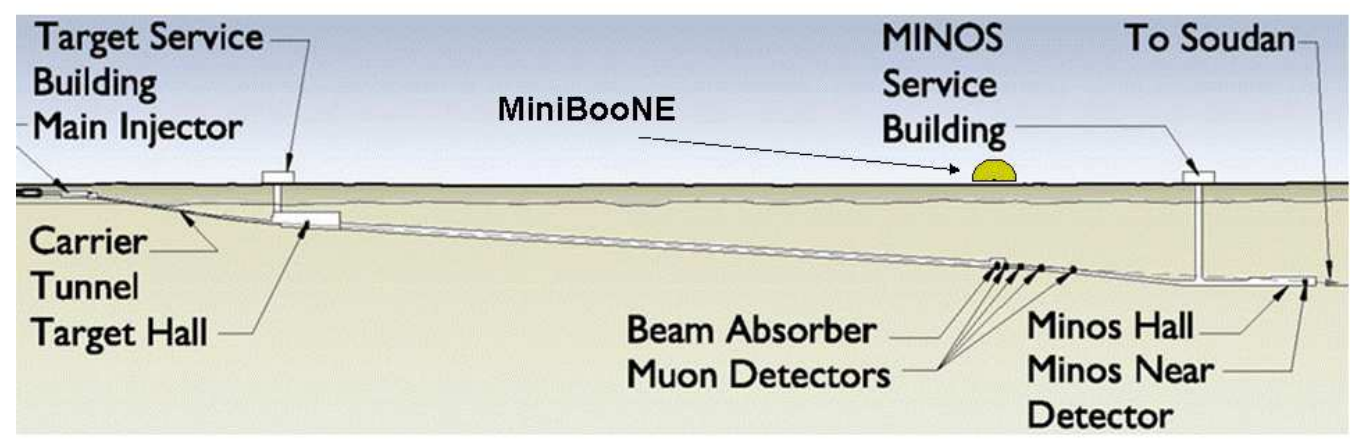

NuMI event composition at MB

$v_{\mu}-81 \%, v_{e}-5 \%, \bar{v}_{\mu}-13 \%, \bar{v}_{e}-1 \%$

Figure 21: The NuMI beam.

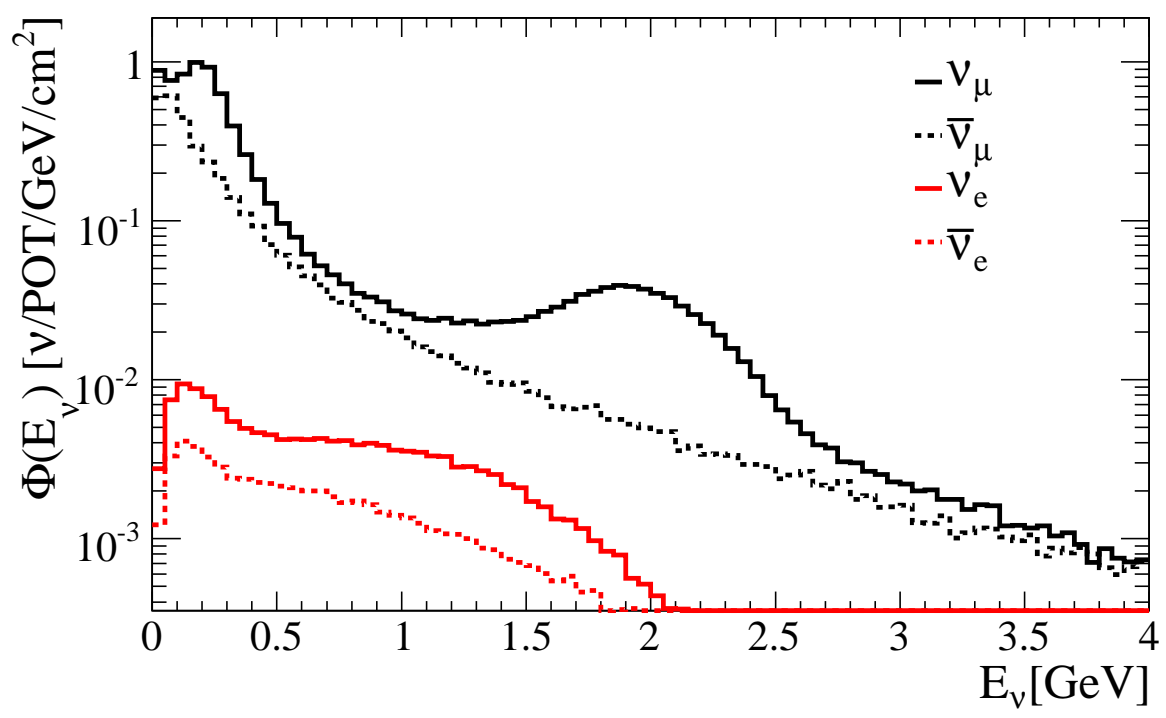

Figure 22: The estimated neutrino flux at the MiniBooNE detector from the NuMI beam. 


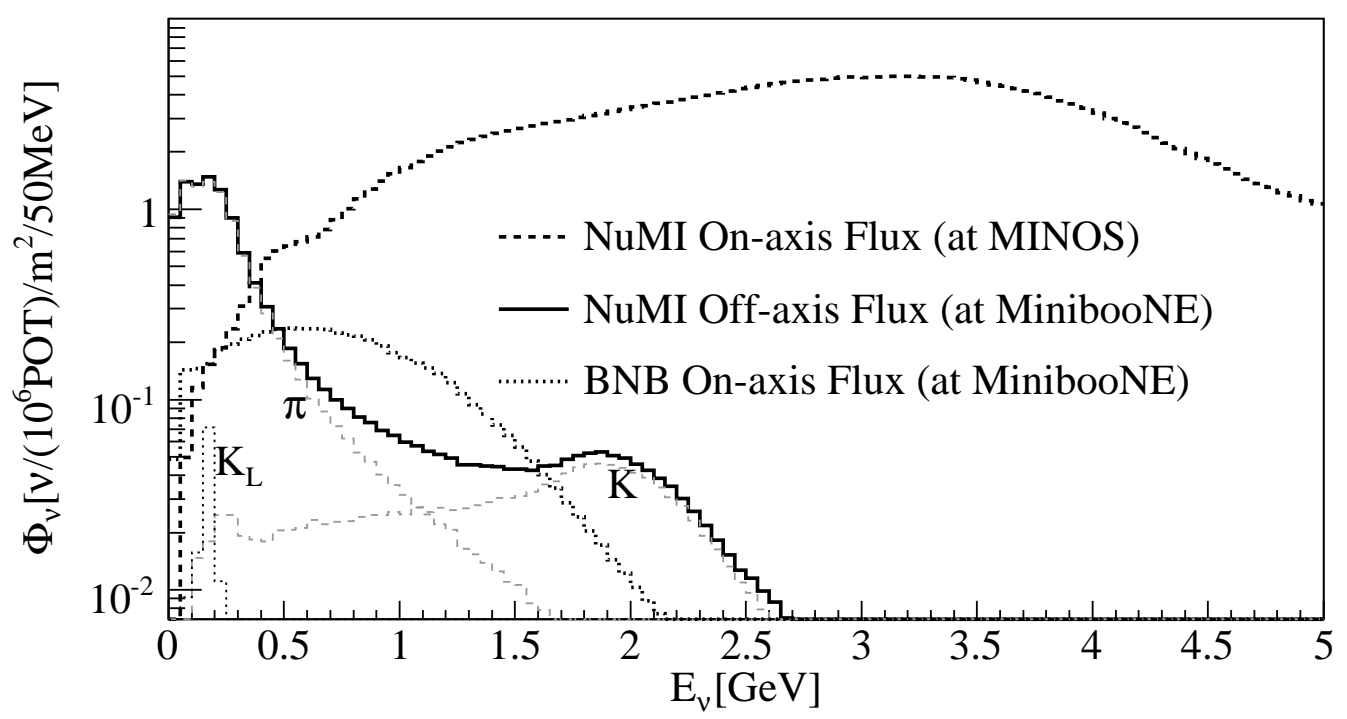

Figure 23: A comparison between the BNB and NuMI neutrino fluxes.

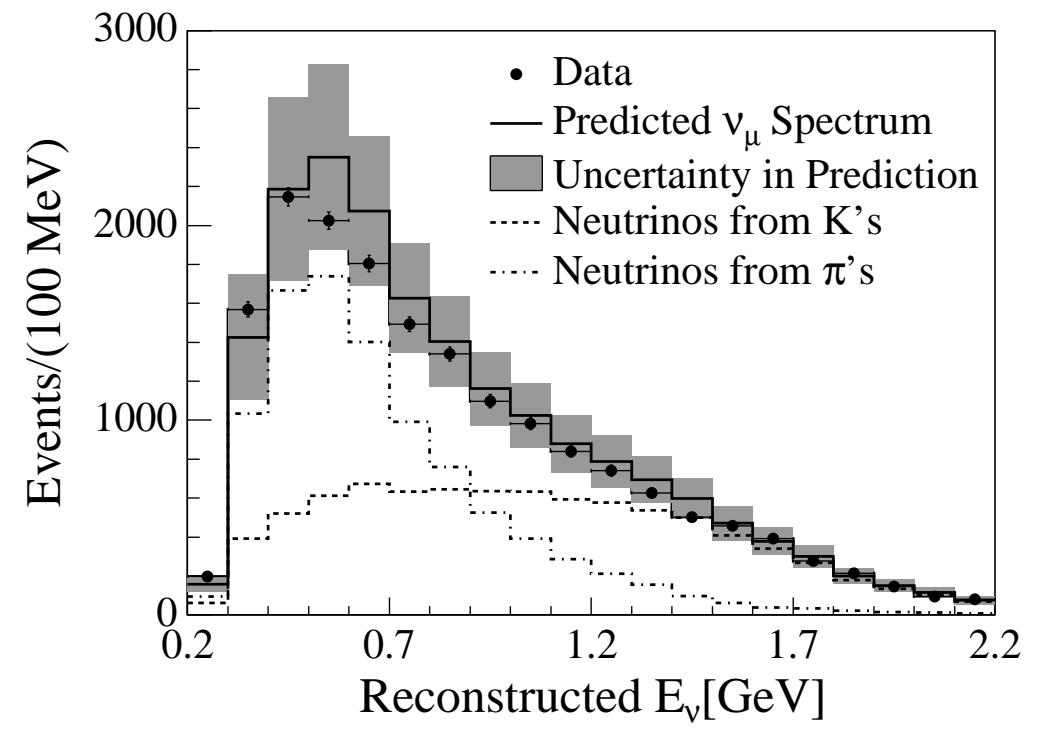

Figure 24: The comparison between data events (points with error bars) and the MC simulation (histogram) for NuMI-induced $\nu_{\mu} C C Q E$ candidate events. 


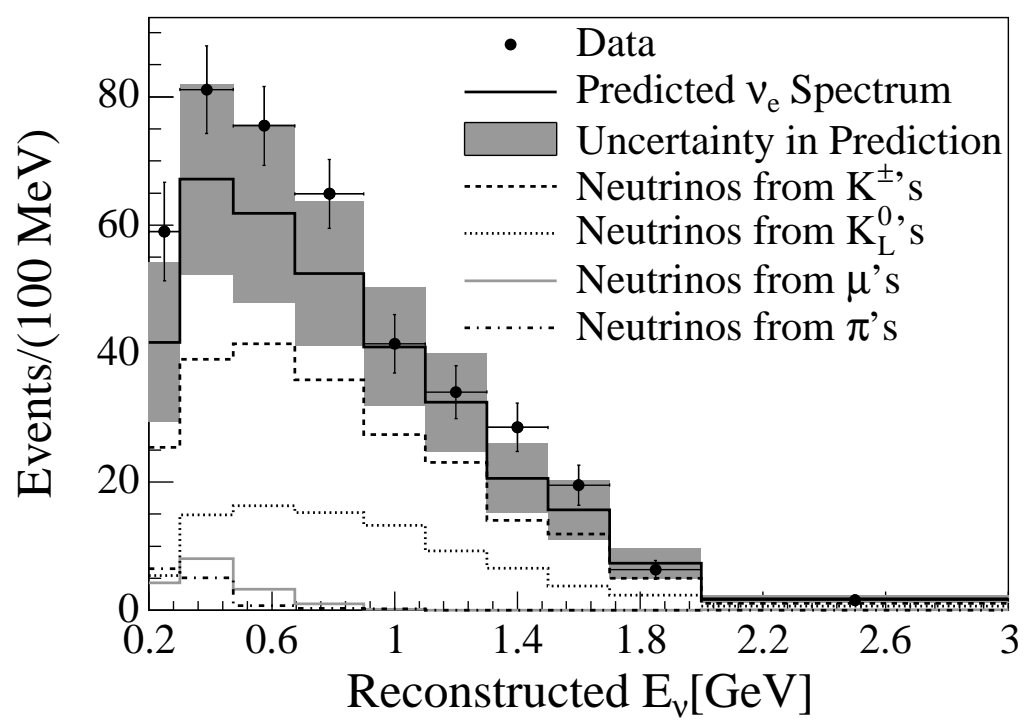

Figure 25: The comparison between data events (points with error bars) and the MC simulation (histogram) for NuMI-induced $\nu_{e} C C Q E$ candidate events.

reveal something important about the nature of the $\nu_{e}$ sample.

\subsection{MiniBooNE Disappearance Results}

MiniBooNE has also searched for $\nu_{\mu}$ and $\bar{\nu}_{\mu}$ disappearance 32 . Fig. 26 shows the sensitivities and limits at 90\% CL for $\nu_{\mu}$ disappearance (top plot) and $\bar{\nu}_{\mu}$ disappearance (bottom plot). The stars on the plots show the best fits in each case: $\Delta m^{2}=17.50 \mathrm{eV}^{2}, \sin ^{2} 2 \theta=0.16$, and $\chi^{2}=12.72 / 14 \mathrm{DF}$ for $\nu_{\mu}$ disappearance and $\Delta m^{2}=31.30 \mathrm{eV}^{2}, \sin ^{2} 2 \theta=0.96$, and $\chi^{2}=5.43 / 14 \mathrm{DF}$ for $\bar{\nu}_{\mu}$ disappearance. The $\chi^{2}$ values for no disappearance oscillations are $\chi^{2}=17.78 / 16 \mathrm{DF}$ and $\chi^{2}=10.29 / 16$ $\mathrm{DF}$, respectively. Improved disappearance sensitivities are expected with the joint SciBooNE/MiniBooNE analysis, which should be completed soon. Note, however, that the joint SciBooNE/MiniBooNE analysis will not be nearly as powerful as the joint BooNE/MiniBooNE analysis due to the small size of SciBooNE and the larger systematic errors from SciBooNE's different detector technology.

\section{BooNE}

The BooNE experiment involves building a second detector at a cost of $\sim \$ 8 \mathrm{M}$ along the BNB at FNAL at a closer distance of $\sim 200 \mathrm{~m}$. With two detectors, many of the systematic errors will cancel, as the neutrino flux varies as $1 / r^{2}$ to good approximation, so that a ratio of events in the two detectors will provide a sensitive search for $\nu_{e}$ and $\bar{\nu}_{e}$ appearance and $\nu_{\mu}$ and $\bar{\nu}_{\mu}$ disappearance. Furthermore, by comparing the rates for a $\mathrm{NC}$ reaction, such as $\mathrm{NC} \pi^{0}$ scattering or NC elastic 


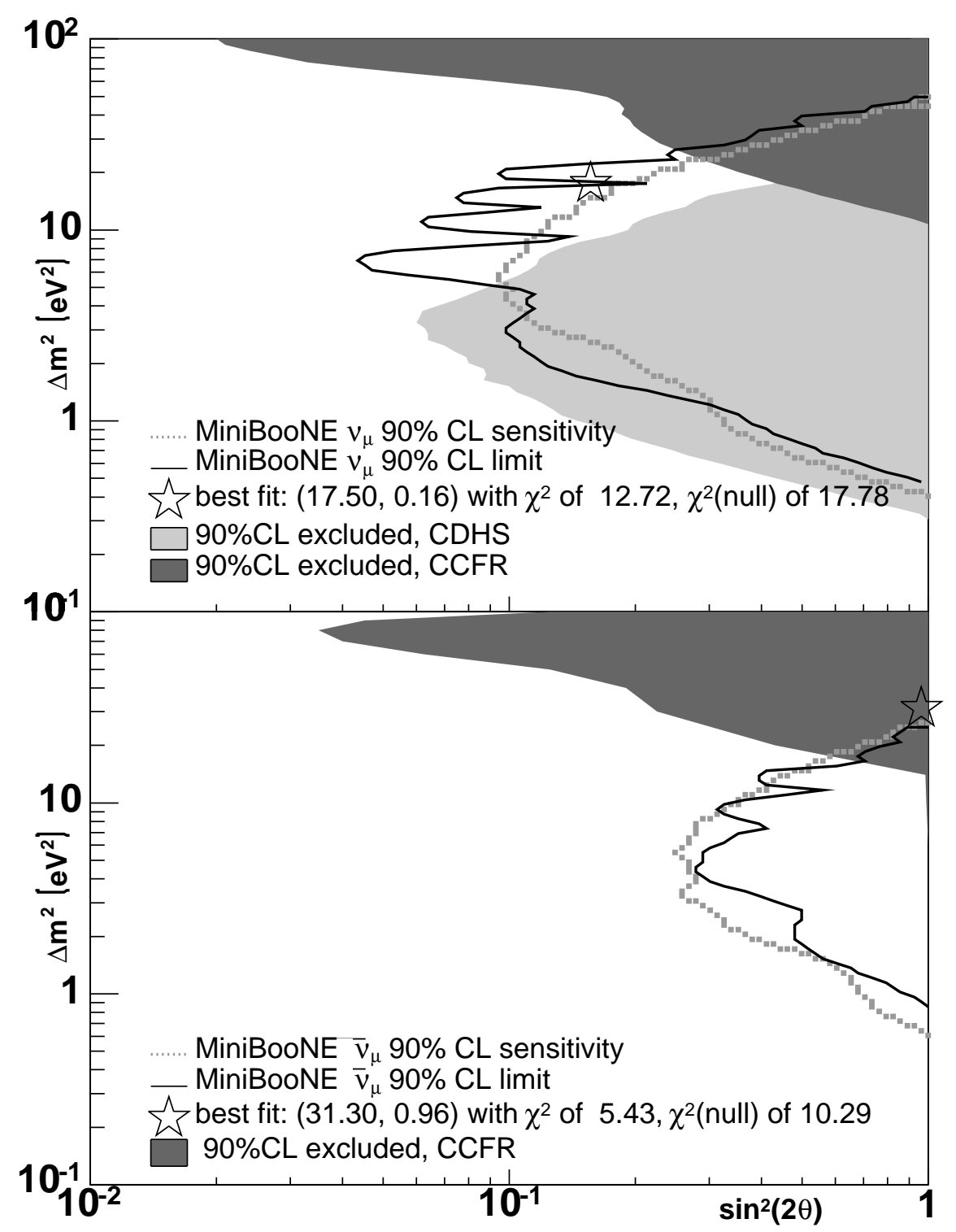

Figure 26: The sensitivities and limits at 90\% CL for $\nu_{\mu}$ disappearance (top plot) and $\bar{\nu}_{\mu}$ disappearance (bottom plot). 
scattering, a direct search for sterile neutrinos can be made. An even cheaper option would be to move the MiniBooNE detector to a different location at a cost of only $\$ 4 \mathrm{M}$. If the MiniBooNE detector were moved to a distance of $200 \mathrm{~m}$ from the neutrino source, then the event rate would increase by a factor of $\sim 7$ due to the dependence of the neutrino flux on distance. An additional advantage of moving MiniBooNE is that MicroBooNE could then move into the original MiniBooNE enclosure and, therefore, save the expense of building a new MicroBooNE enclosure. In either case, after less than a year of running, the comparison of the event rates at the two locations will determine whether the low-energy excess observed by MiniBooNE was due to neutrino oscillations. In addition, $\nu_{\mu}$ and $\bar{\nu}_{\mu}$ disappearance will be searched for with high sensitivity in the $\Delta m^{2}>0.1 \mathrm{eV}^{2}$ mass region, and LSND antineutrino oscillations can be tested directly by searching for $\bar{\nu}_{e}$ appearance. By comparing neutrino oscillations to antineutrino oscillations, BooNE will be able to search for $\mathrm{CP}$ and $\mathrm{CPT}$ violation in the lepton sector at short baseline $\left(\Delta m^{2}>0.1 \mathrm{eV}^{2}\right)$. For the sensitivities discussed below, it is assumed that the near detector will run for $\sim 1 \mathrm{E} 20 \mathrm{POT}$ in both neutrino mode and antineutrino mode.

\subsection{Fluxes and Event Rates}

This section gives a detailed comparison of the expected neutrino fluxes at the near (200 meter) and far (541 meters) positions. In the Booster neutrino beam (BNB), the primary beam is produced by the $8 \mathrm{GeV}$ Fermilab's rapid-cycling $(15 \mathrm{~Hz})$ booster accelerator, which produces $1.6 \mu \mathrm{s}$ batches of protons each containing around $4.5 \times 10^{12}$ protons.

At that primary proton energy, there are only four significant species of neutrinos: $\nu_{\mu}$ and $\bar{\nu}_{\mu}(\sim 99.5 \%)$, and a small contamination $(\sim 0.5 \%)$ of $\nu_{e}$ and $\bar{\nu}_{e}$. There are two primary parent components to the fluxes: neutrinos from charged pion decays and neutrinos from kaon decays. The $K^{+}$component dominates the $\nu_{\mu}$ spectrum above neutrino energies of $2.5 \mathrm{GeV}$, where a clear break is observed in the slope of the energy spectrum. The $\bar{\nu}_{\mu}$ spectra are mainly from charged pion decay, and the $\nu_{e}$ and $\bar{\nu}_{e}$ spectra are composed of two parts, muon decays and kaon decays.

The standard MiniBooNE Geant4 based beam simulation and decay program packages were used to generate fluxes 27 . Those packages include the transport of muon polarization (neglecting $g-2$ precession effects) and appropriate form factors in leptonic kaon decays. The primary production of pions by $8 \mathrm{GeV}$ protons was measured by the HARP experiment $\frac{33}{3}$ and is used as input in the simulation, while secondary interactions in the beam line are handled by standard Geant4 physics packages.

The fluxes shown here represent the spectrum of neutrinos that intersect a sphere of radius $610.6 \mathrm{~cm}$, positioned at either the near or far location. The fluxes are "unoscillated" and therefore have only $\nu_{\mu}\left(\bar{\nu}_{\mu}\right)$ and $\nu_{e}\left(\bar{\nu}_{e}\right)$ components. No matter effects in propagating the neutrinos to the detector are included, as they are expected to be small in the standard, 3-generation, active neutrino model $(\mathrm{S} \nu \mathrm{M})$. 
Figs. 27, 28, 29, and 30 show the fluxes for the four neutrino species at the near and far locations, for both neutrino mode and antineutrino mode. Table 6 gives the same fluxes, integrated over neutrino energy, while Table 7 gives the average neutrino energy in each case.

In neutrino mode, the $\nu_{\mu}$ flux near/far ratio is 7.5. Most of the near far ratios are between 7.0 and 8.0. Another characteristic of the near/far flux comparisions is that the average energy of the neutrinos in the near position is between 5 and 10 percent less than the corresponding average energy in the far position. This lower energy is expected since the near detector has a larger angular acceptance with respect to the neutrino target.

Fig. 31 shows the energy distribution for $\nu_{\mu}$ CCQE events at the near $\left(1.0 \times 10^{20}\right.$ $\mathrm{POT})$ and far $\left(6.462 \times 10^{20} \mathrm{POT}\right)$ locations for neutrino mode. The spectral differences are again due to the larger angular acceptance of the near detector. That larger decay angle translates to lower neutrino energies in the near detector, typically $\sim 10 \%$ lower in the 200/541 meter comparison. This extrapolation is relatively straight forward as the angular divergence of the daughter neutrinos in the decays is much larger than the angular divergence of the decaying mesons themselves. For example, even at 3 $\mathrm{GeV}$, daughter neutrinos from pion and kaon decays will have opening angles of $50 \mathrm{mrad}$ and $\sim 150 \mathrm{mrad}$, respectively, while the allowed angular divergence of the beam tunnel is only $\sim 20 \mathrm{mrad}$.

Because of the nearly complete overlap in decay particle phase space that contributes to neutrinos in the near and far positions, we expect that uncertainties in the flux prediction will largely cancel when comparing the two event rates from the near and far positions. As systematic errors introduced by uncertainties in the detector efficiency and neutrino cross section will also largely cancel, the comparison of the two positions will allow a much-needed, accurate measurement of non-S $\nu \mathrm{M}$ neutrino oscillation effects in the $\Delta m^{2}$ range of $0.1-10 \mathrm{eV}^{2}$.

Table 6: Integrated fluxes per POT for the various species of neutrinos at the near and far positions, for both neutrino mode and antineutrino mode.

Fluxes $\nu /\left(\mathrm{cm}^{2} P O T\right)$

\begin{tabular}{|c|c|c|c|c|}
\hline & \multicolumn{2}{|c|}{$\nu$ mode } & \multicolumn{2}{c|}{$\bar{\nu}$ mode } \\
\hline$\nu$ species & Near & Far & Near & Far \\
\hline$\nu_{\mu}$ & $7.49 \times 10^{-8}$ & $1.03 \times 10^{-8}$ & $8.12 \times 10^{-9}$ & $1.08 \times 10^{-9}$ \\
$\bar{\nu}_{\mu}$ & $5.20 \times 10^{-9}$ & $6.52 \times 10^{-10}$ & $4.30 \times 10^{-8}$ & $5.77 \times 10^{-9}$ \\
$\nu_{e}$ & $4.50 \times 10^{-10}$ & $5.74 \times 10^{-11}$ & $9.5 \times 10^{-11}$ & $1.34 \times 10^{-11}$ \\
$\bar{\nu}_{e}$ & $4.61 \times 10^{-11}$ & $6.00 \times 10^{-12}$ & $2.00 \times 10^{-10}$ & $2.53 \times 10^{-11}$ \\
\hline
\end{tabular}

\subsection{Possible Scenarios for a Near Detector}

The MiniBooNE detector has operated at a location of 541 meters from the 
Table 7: Average neutrino energies for the various species of neutrinos at the near and far positions, for both neutrino mode and antineutrino mode.

Average $\nu$ energies $(\mathrm{MeV})$
\begin{tabular}{|c|c|c|c|c|}
\hline & \multicolumn{2}{|c|}{$\nu$ mode } & \multicolumn{2}{c|}{$\bar{\nu}$ mode } \\
\hline$\nu$ species & Near & Far & Near & Far \\
\hline$\nu_{\mu}$ & 721 & 807 & 631 & 703 \\
$\bar{\nu}_{\mu}$ & 412 & 461 & 593 & 649 \\
$\nu_{e}$ & 903 & 957 & 856 & 874 \\
$\bar{\nu}_{e}$ & 917 & 971 & 677 & 716 \\
\hline
\end{tabular}

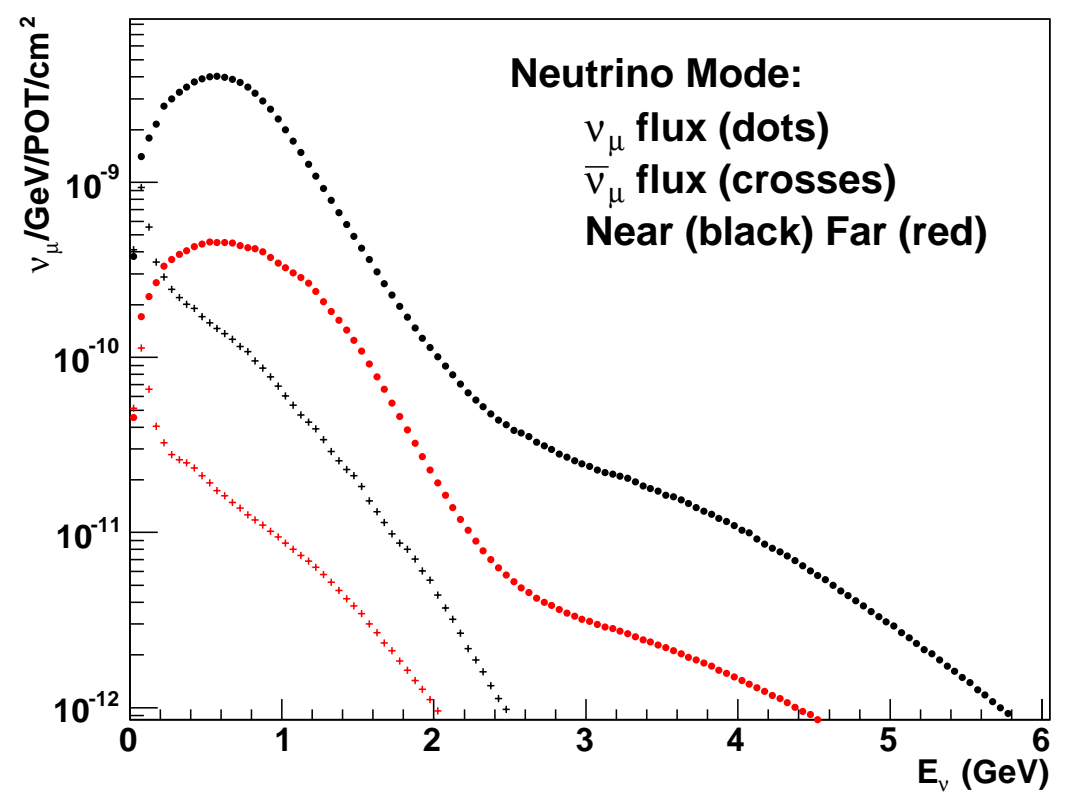

Figure 27: The $\nu_{\mu}$ and $\bar{\nu}_{\mu}$ fluxes at both the near and far locations in neutrino mode.

Booster Neutrino Beam (BNB) target since September 1, 2002. The primary purpose of the experiment was to search for the transmutation, or oscillation, of muon neutrinos into electron neutrinos as they travel the $\sim 525$ meters to the detector. The BNB was designed to produce a nearly pure beam of $\nu_{\mu}$, which provides an ideal setting to look for excess $\nu_{e}$ events. While the most sensitive neutrino oscillation experiments are two detector systems, which afford a comparison of similar detectors at two different distances, MiniBooNE was built as a single detector system in order to reduce costs. It was felt that the systematic error incurred by not building a second detector could be overcome by using internal measurements in the single detector. As $\nu_{\mu}$ were not expected to oscillate significantly, it was planned to use their rate as a normalization for $\nu_{e}$ interactions, thus constraining backgrounds to $\nu_{e}$ events from 


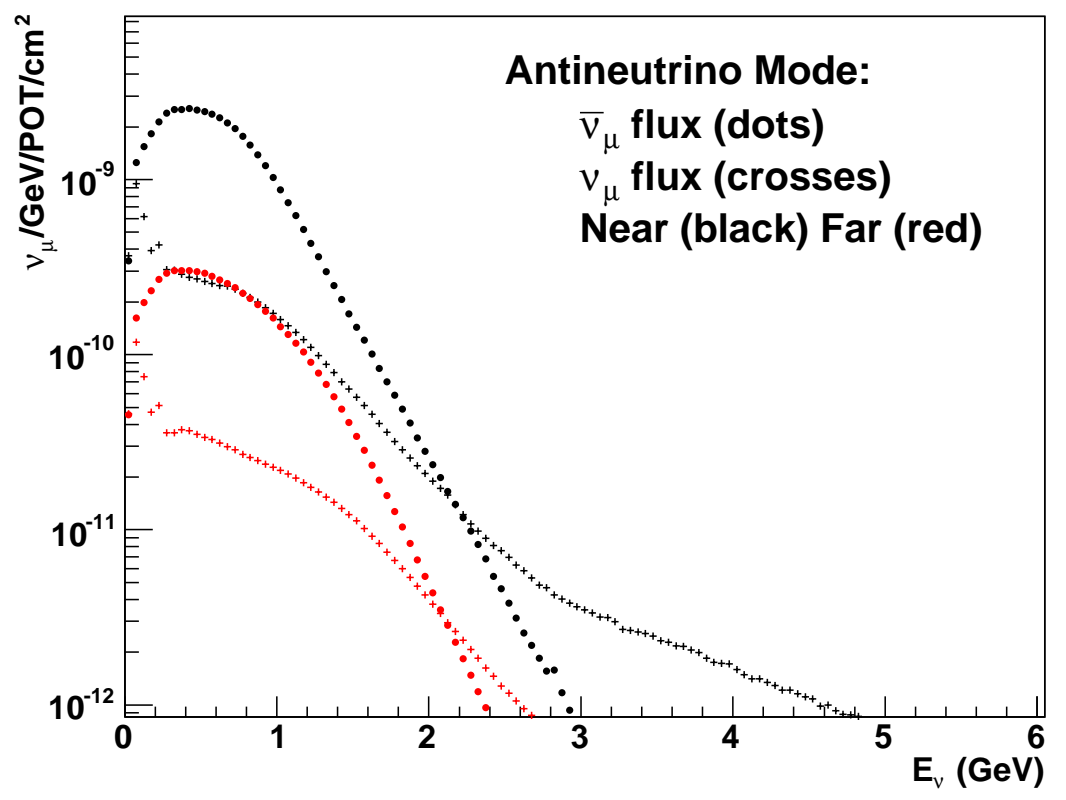

Figure 28: The $\nu_{\mu}$ and $\bar{\nu}_{\mu}$ fluxes at both the near and far locations in antineutrino mode.

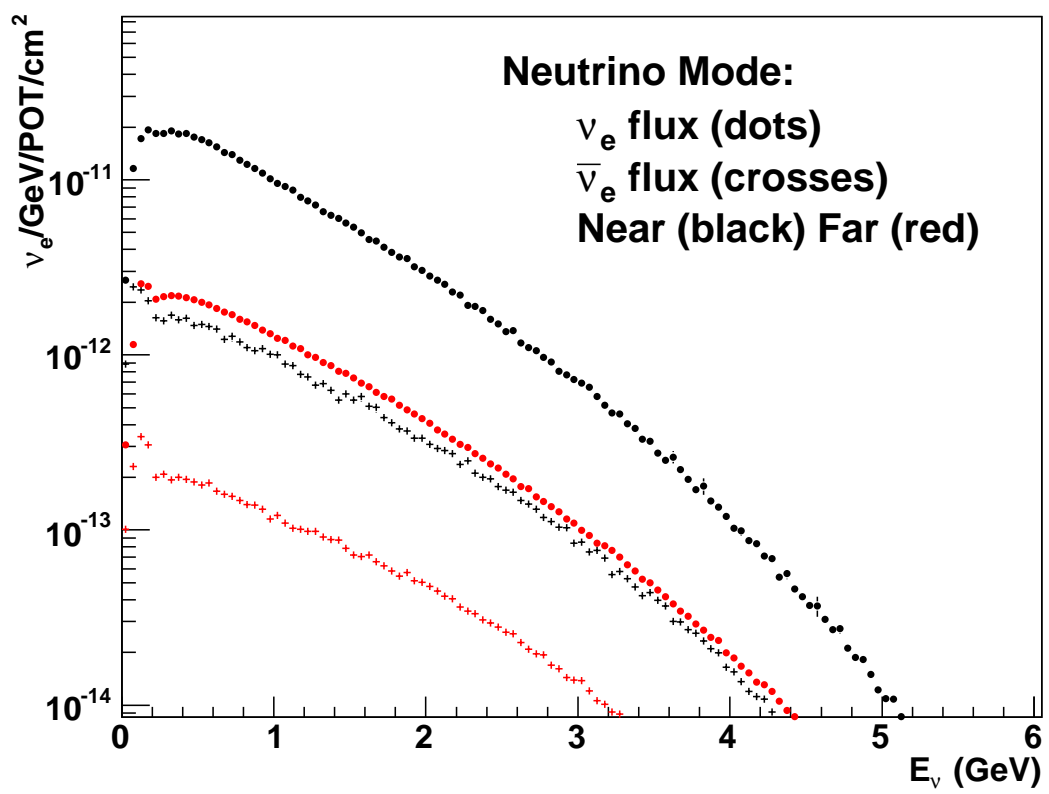

Figure 29: The $\nu_{e}$ and $\bar{\nu}_{e}$ fluxes at both the near and far locations in neutrino mode.

oscillations.

The MiniBooNE proposal foresaw that a second detector at a different distance 


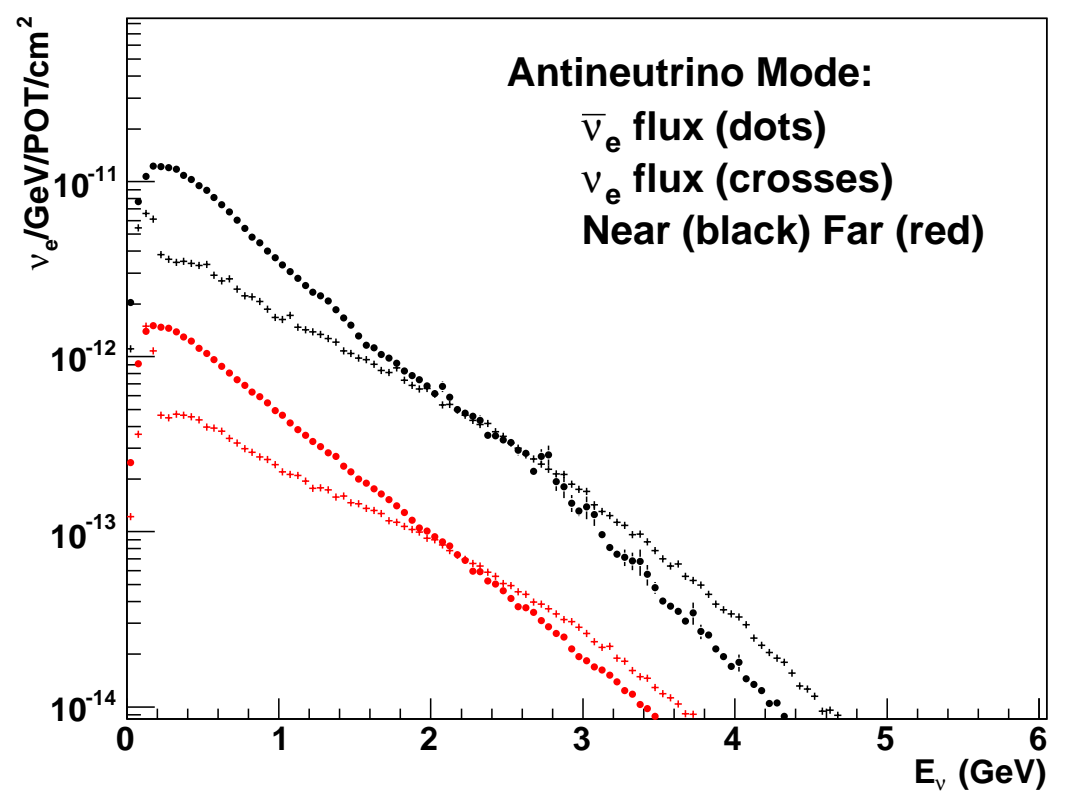

Figure 30: The $\nu_{e}$ and $\bar{\nu}_{e}$ fluxes at both the near and far locations in antineutrino mode.

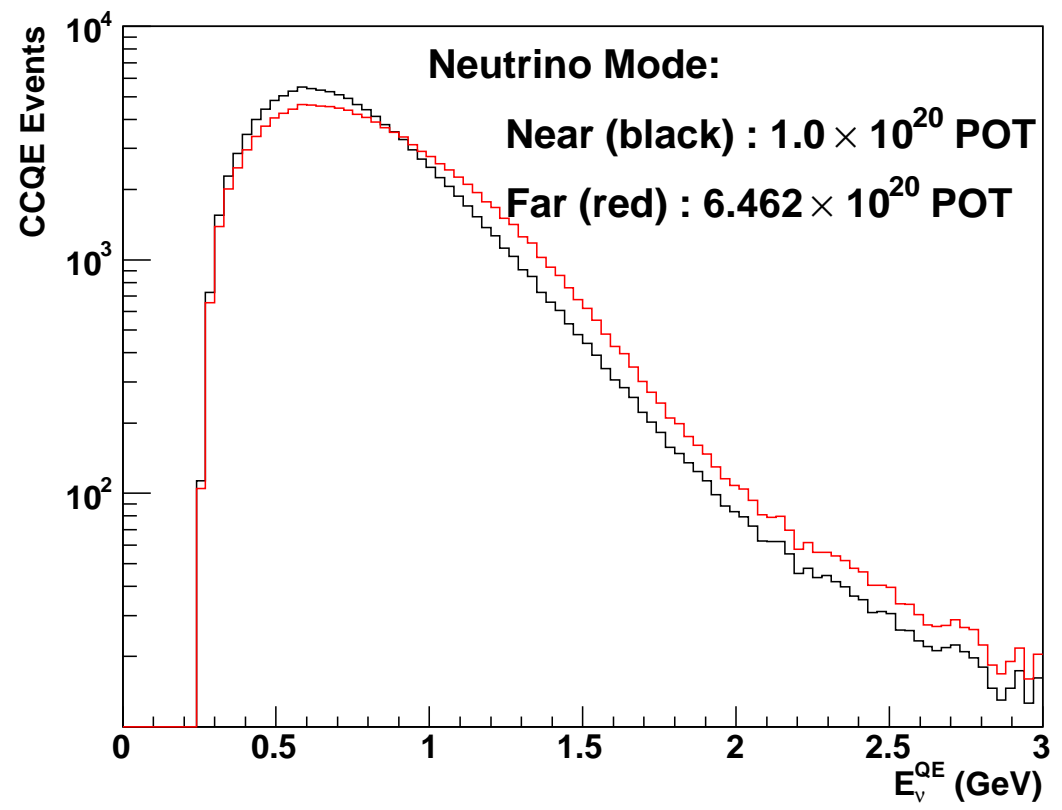

Figure 31: The distribution of energies for reconstructed $\nu_{\mu} C C Q E$ events, at the near location with $1.0 \times 10^{20}$ POT and at the far location with the current $6.462 \times 10^{20}$ POT. 
would be required to ascertain the nature of the signal, if a significant signal were observed in the single-detector setup. A second detector, located at a different distance from the BNB target could potentially remove the large systematic errors that would complicate the interpretation of the MiniBooNE data.

There are several possible routes to a more precise measurement which need to be considered: a new detector could be constructed at a near position; similarly, a new detector could be constructed at a far position; and, it was recently realized that the MiniBooNE detector could be relocated to a new position at either a near or a far location. Each of those possibilities has advantages and disadvantages.

4.2.1.

Near or Far?

The choice between constructing a near detector at $\sim 200$ meters and a far detector at $\sim 1000$ meters can be made based on expediency. For $\Delta m^{2}<2 \mathrm{eV}^{2}$, a near detector will not see a large signal directly but can be used to accurately measure the expected backgrounds to any possible oscillation signal in the far detector. Those backgrounds, in both appearance and disappearance measurements, can be measured at $\sim 7-8$ times the rate that the MiniBooNE detector accumulated data. Thus a sample of neutrinos with statistics equivalent to MiniBooNE's existing data set of $6.462 \times 10^{20}$ POT will only require $\sim 1.0 \times 10^{20} \mathrm{POT}$ and yield an $\sim 5 \sigma$ result. An identical far detector, on the other hand, would also yield an $\sim 5 \sigma$ result with $\sim 1.0 \times 10^{20}$ POT, however the signal would only be $\sim 20$ events on top of a background of $\sim 16$ events. An unsatisfying, ambiguous result could occur with such low statistics.

4.2 .2 .

\section{Moving MiniBooNE}

Relocating the MiniBooNE detector to a near position $\sim 200$ meters from the target, shown in Fig. 32, will likely be the least costly option. There are a number of potential advantages to this approach. The detector is already built so that the cost of constructing a new detector is avoided. The neutrino flux at 200 meters will be 7-8 times larger, so that the time needed to accumulate a data sample equivalent to the existing sample will be less than one year.

The comparison of measurements taken by the same detector operated at two locations will be free of systematic errors associated with neutrino-nucleus cross sections and detector response. The comparison should clearly reveal the nature of the MiniBooNE excess. The 541/200 meter data comparison will also allow MiniBooNE to measure $\nu_{\mu}$ and $\bar{\nu}_{\mu}$ disappearance at the few percent level.

The detector relocation could take place in two ways: transporting the existing detector and electronics to the new location, or building a new detector at the new location using parts from the existing detector. In both cases the MiniBooNE dectector would be drained of oil and the oil stored in rail cars or a separate oil storage 


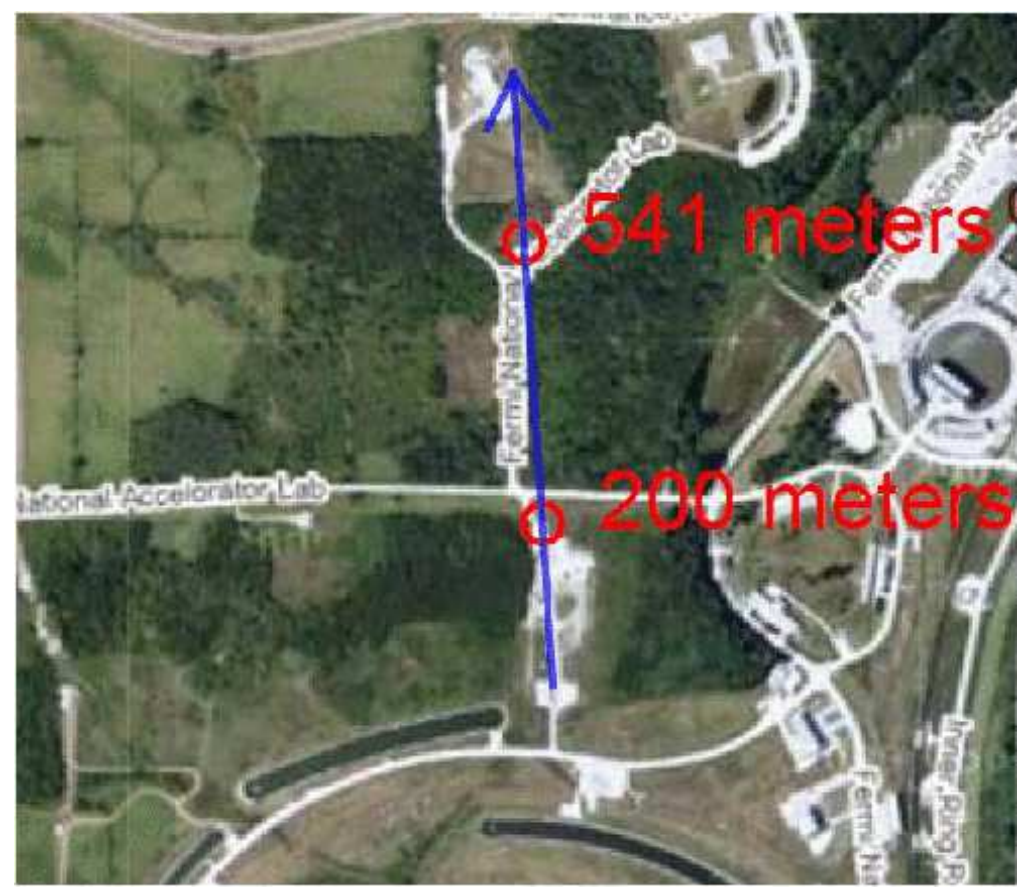

Figure 32: An arial view of Fermilab showing a possible location of a near detector at $\sim 200$ meters. The blue arrow indicates the direction of the booster neutrino beam.

tank. With the mineral oil removed, the detector weighs only $\sim 80$ tons, and could be transported whole to the near site. Mobile cranes with 80 ton lfting capacities are readily available commercially at a reasonable cost.

\subsection{3.}

\section{A New Detector}

An alternative to relocating the existing MiniBooNE detector and its electronics is to construct a new sperical tank at the near position and remove PMTs by hand from the old tank. The new tank would be constructed by repeating the constuction effort made for MiniBooNE. In either case, one would re-use the existing, albeit old, LSND electronics currently used by MiniBooNE. The cost of either case is estimated to be $\sim \$ 4 \mathrm{M}$.

The most desirable, and most costly, option is to construct an entirely new detector at the near location. This would require more time because new electronics would have to be developed, a new oil mineral supplier found, and new phototubes purchased. The lead times on those items would be about two years. The cost for that effort is estimated to be $\sim$ twice that of moving the existing detector, $\sim \$ 8 \mathrm{M}$.

It is not yet understood how the systematic error in detector response will translate between the old MiniBooNE detector and the newly constructed detector, since it will have different oil, PMTs, and electronics. Nevertheless, choosing to construct an 
Table 8: The expected excess of low-energy events $\left(200<E_{\nu}<475 \mathrm{MeV}\right)$ in antineutrino mode (1E21 POT) for various hypotheses, assuming a 2.5\% systematic error and assuming that the neutrino low-energy excess is correct. Also shown is the significance of the excesses.

\begin{tabular}{|c|c|c|}
\hline Hypothesis & Expected Excess of $\bar{\nu}$ Events & Significance \\
\hline Same $\sigma$ & $111.6 \pm 13.5 \pm 4.6$ & $7.8 \sigma$ \\
$\pi^{0}$ Scaled & $58.2 \pm 13.5 \pm 4.6$ & $4.1 \sigma$ \\
POT Scaled & $202.6 \pm 13.5 \pm 4.6$ & $14.2 \sigma$ \\
BKGD Scaled & $62.8 \pm 13.5 \pm 4.6$ & $4.4 \sigma$ \\
CC Scaled & $61.2 \pm 13.5 \pm 4.6$ & $4.3 \sigma$ \\
Kaon Scaled & $119.2 \pm 13.5 \pm 4.6$ & $8.4 \sigma$ \\
Neutrino Scaled & $20.2 \pm 13.5 \pm 4.6$ & $1.4 \sigma$ \\
\hline
\end{tabular}

entirely new detector would allow for simultaneous operation of both the near and far detector and eliminate any fear, unfounded or not, that the neutrino beam had changed in character.

Ideally, the near BooNE detector would have the same dimensions as the MiniBooNE detector in order to reduce systematic uncertainties. However, another possibility would be to build a smaller detector $(\sim 8 \mathrm{~m}$ diameter $)$ at a lower cost $(\sim \$ 4 \mathrm{M})$ if systematic errors were estimated to be sufficiently small.

\subsection{Testing the Low-Energy Excess}

BooNE will be able to determine whether the low-energy excess is due to neutrino oscillations and will be able to test various hypotheses by comparing the low-energy excesses in neutrino and antineutrino modes. If the low-energy excess is due to background, then the near detector will observe the same relative excess as the far detector. If the excess is due to neutrino oscillations at low $\Delta m^{2}$, then no low-energy excess will be observed in the near detector and the current low-energy excess in the far detector, assuming a $2.5 \%$ systematic error, will equal to $128.8 \pm 20.4 \pm 10.4$ events $(5.6 \sigma)$. For testing various hypotheses, Table 8 shows the expected excess of low-energy events $\left(200<E_{\nu}<475 \mathrm{MeV}\right)$ in antineutrino mode for 1E21 POT, assuming a $2.5 \%$ systematic error and assuming that the neutrino excess is correct. Also shown is the significance of the excesses. By comparing the measured excess in the antineutrino data to the expected excesses, the different hypotheses can be shown to be either consistent with data or ruled out.

\section{4. $\nu_{e}$ and $\bar{\nu}_{e}$ Appearance}

The sensitivities for $\nu_{e}$ and $\bar{\nu}_{e}$ appearance were obtained by using the MiniBooNE Monte Carlo simulation, assuming statistical errors with the expected MiniBooNE statistics (6.5E20 POT in neutrino mode and 1E21 POT in antineutrino mode) and a 
full error matrix with correlated and uncorrelated systematic errors. Also, we assume 2E20 POT in the near detector, equally divided between neutrino and antineutrino modes. Fig. 33 shows the estimated sensitivity for $\nu_{e}$ appearance for $E_{\nu}>200$ $\mathrm{MeV}$. Although simple two-neutrino oscillations have already been ruled out as an explanation of the LSND signal, it is interesting that the full LSND region is covered at the approximately $5 \sigma$ level. Therefore, we would be able to determine whether or not the MiniBooNE low-energy excess is due to a more complicated oscillation mechanism at the $\sim 1 \mathrm{eV}^{2}$ scale. Fig. 34 shows the estimated sensitivity for $\bar{\nu}_{e}$ appearance, where we assume that the error matrix is the same as for neutrinos. The sensitivity is worse than the $\nu_{e}$ appearance sensitivity due to the lower statistics and higher wrong-sign background in antineutrino mode; however, BooNE will still be able to cover the full LSND region at $90 \% \mathrm{CL}$ and provide a direct test of LSND antineutrino oscillations.

\section{5. $\nu_{\mu}$ and $\bar{\nu}_{\mu}$ Disappearance}

The sensitivities for $\nu_{\mu}$ and $\bar{\nu}_{\mu}$ disappearance were obtained by using the MiniBooNE Monte Carlo simulation, assuming statistical errors with the expected MiniBooNE statistics (6.5E20 POT in neutrino mode and 1E21 POT in antineutrino mode) and a full error matrix with correlated and uncorrelated systematic errors. Also, we assume 2E20 POT in the near detector, equally divided between neutrino and antineutrino modes. Fig. 35 shows the estimated sensitivity for $\nu_{\mu}$ disappearance for $E_{\nu}>200 \mathrm{MeV}$. A sensitivity of $\sim 3 \%$ at $90 \% \mathrm{CL}$ is obtained for $\Delta m^{2} \sim 1 \mathrm{eV}^{2}$. In order to see how a signal would appear, Fig. 36 shows the allowed region for $\nu_{\mu}$ disap-

pearance at the global antineutrino best-fit point from reference ${ }^{12}$ : $\Delta m_{14}^{2}=0.915$ $\mathrm{eV}^{2}$ and $\sin ^{2} 2 \theta_{\mu \mu}=0.35$. Figs. 37 and 38 show the correponding limits and allowed regions for $\bar{\nu}_{\mu}$ disappearance, assuming no $\nu_{\mu}$ disappearance and the same error matrix as for neutrinos. The $\bar{\nu}_{\mu}$ sensitivity is slightly worse than the $\nu_{\mu}$ sensitivity due to the lower antineutrino statistics and the $\sim 1 / 3$ wrong-sign $\nu_{\mu}$ component in antineutrino mode. A difference between $\nu_{\mu}$ and $\bar{\nu}_{\mu}$ disappearance would be evidence for CPT violation or effective CPT violation 2699 .

\subsection{Sterile Neutrino Search}

If $\nu_{\mu}$ or $\bar{\nu}_{\mu}$ disappearance is observed, then the $\mathrm{NC} \pi^{0}$ and $\mathrm{NC}$ Elastic reactions can be used to determine whether the disappearance is due to oscillations into active or sterile neutrinos. Oscillations into sterile neutrinos will result in a suppression of events in the far detector, while oscillations into active neutrinos will result in no suppression. Due to the high statistics of the NC $\pi^{0}$ and NC Elastic event samples, the statistical error will be small compared to the systematic error. The sensitivity at $90 \% \mathrm{CL}$ for oscillations into sterile neutrinos at $\Delta m^{2} \sim 1 \mathrm{eV}^{2}$ is estimated to be 


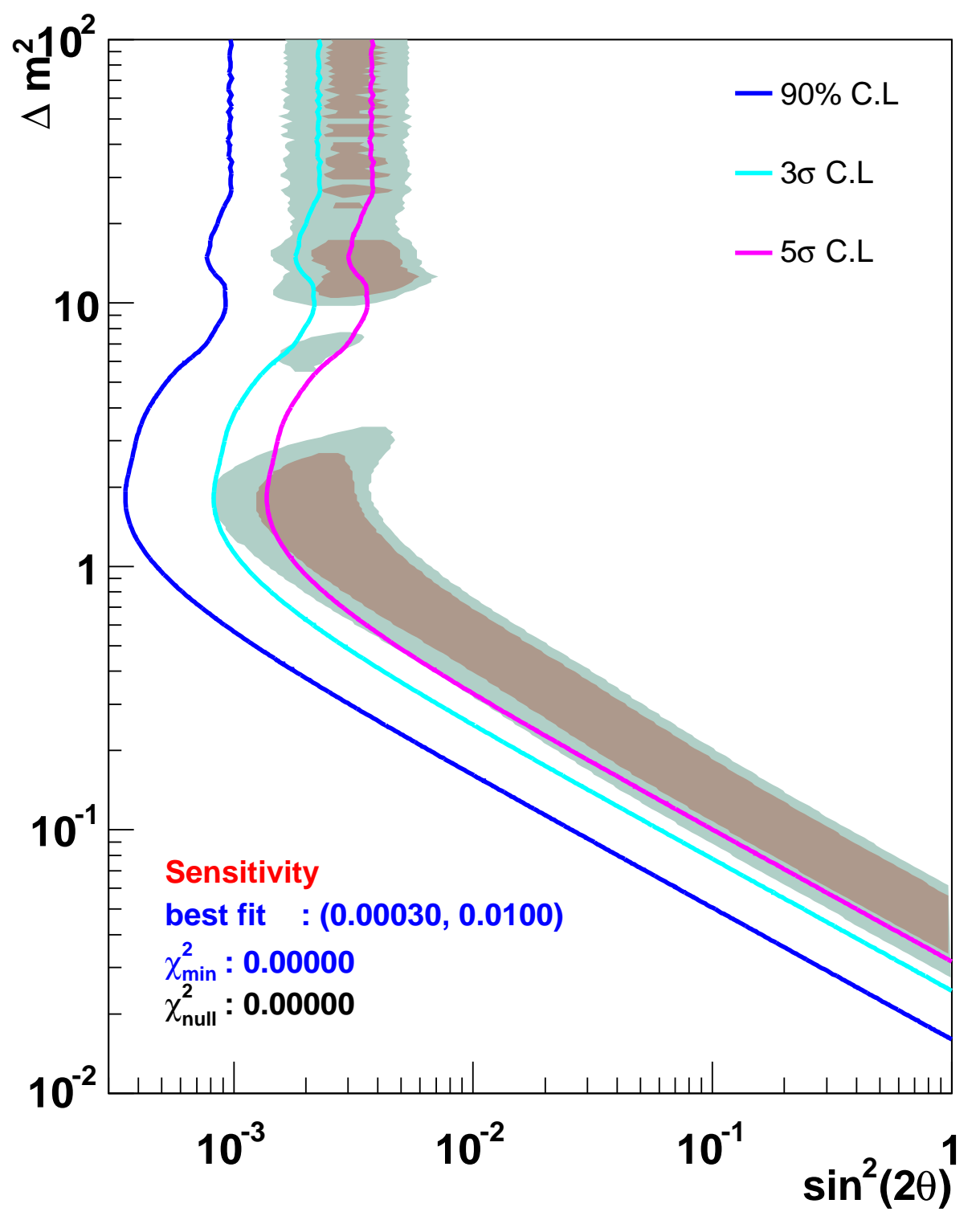

Figure 33: The estimated BooNE sensitivity for $\nu_{e}$ appearance. 


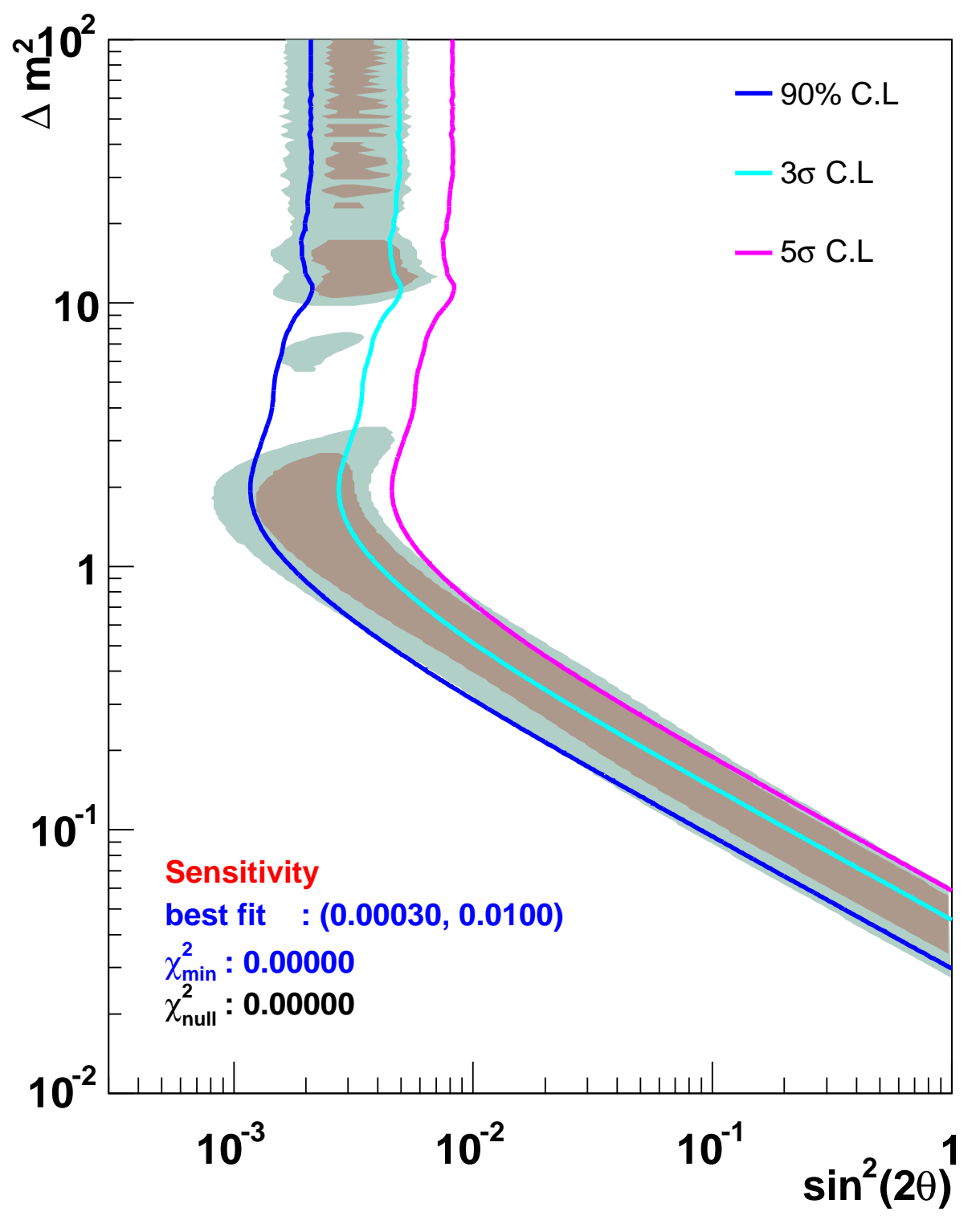

Figure 34: The estimated BooNE sensitivity for $\bar{\nu}_{e}$ appearance. 


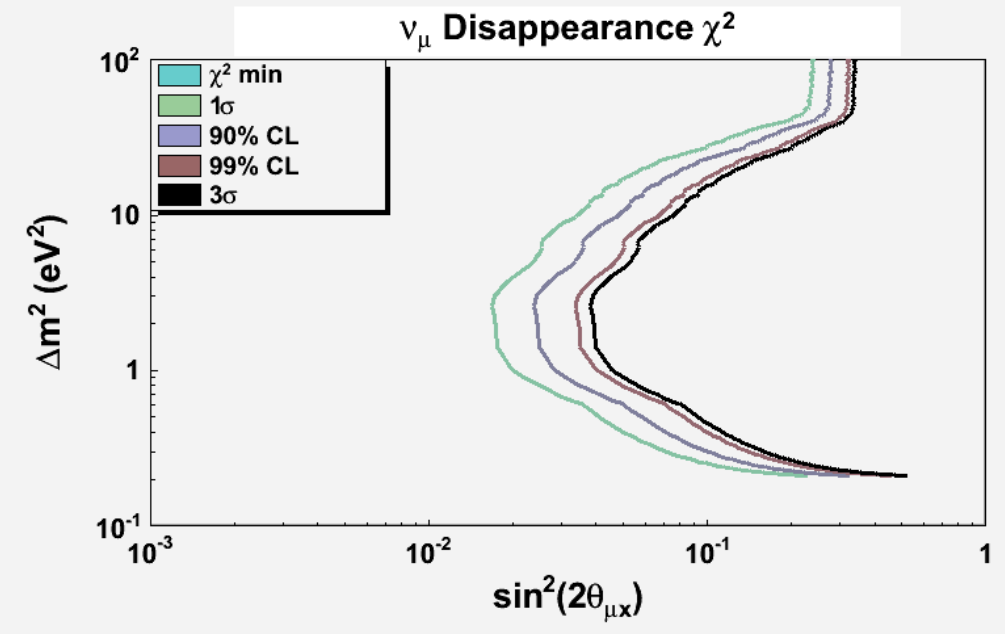

Figure 35: The estimated BooNE sensitivity for $\nu_{\mu}$ disappearance.

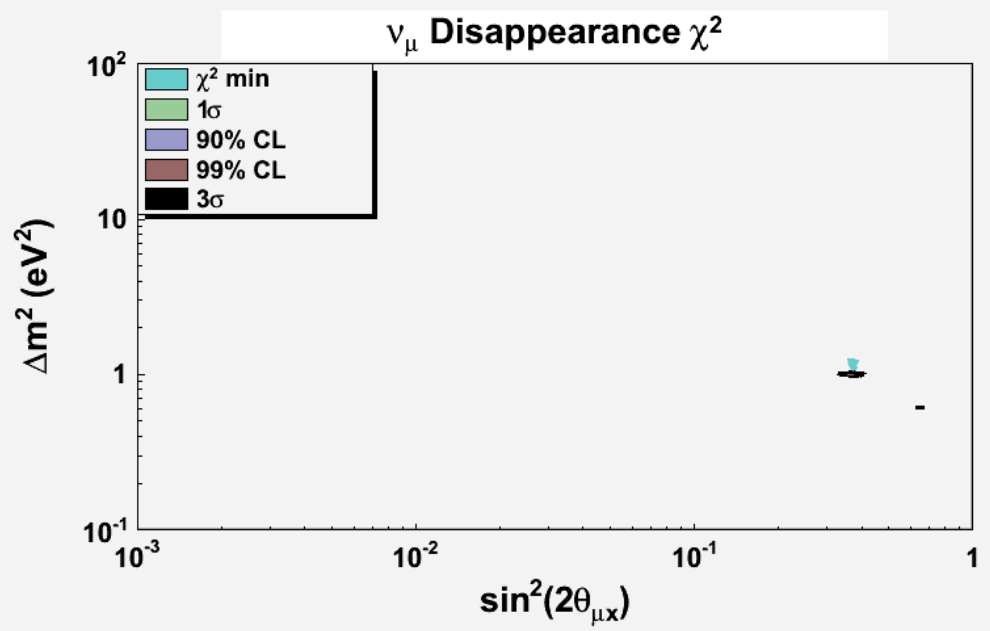

Figure 36: The BooNE allowed region for $\nu_{\mu}$ disappearance at the global antineutrino best-fit point $\Delta m_{14}^{2}=0.915 \mathrm{eV}^{2}$ and $\sin ^{2} 2 \theta_{\mu \mu}=0.35$. 


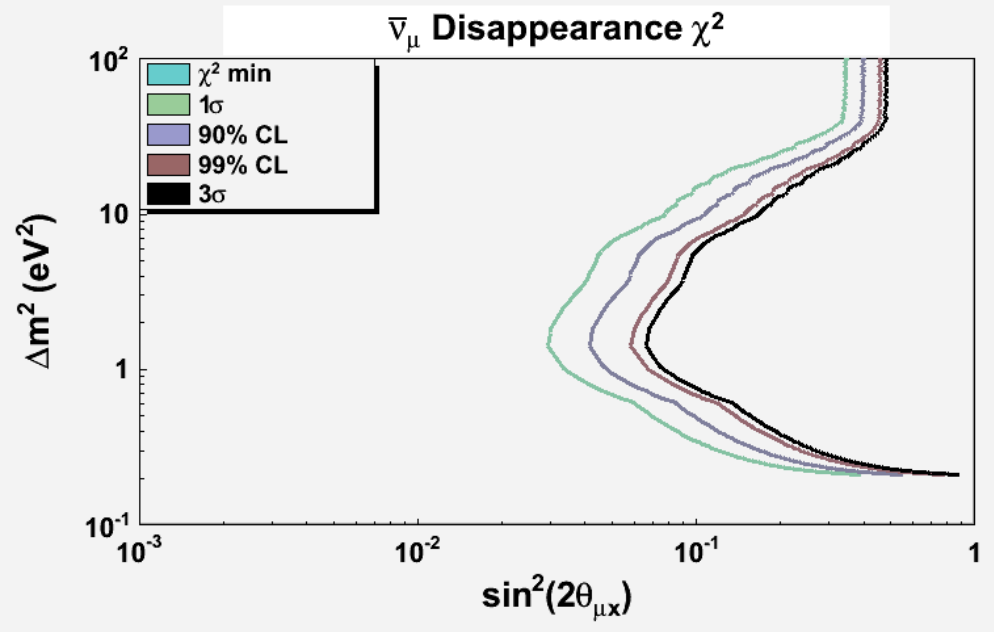

Figure 37: The estimated BooNE sensitivity for $\bar{\nu}_{\mu}$ disappearance.

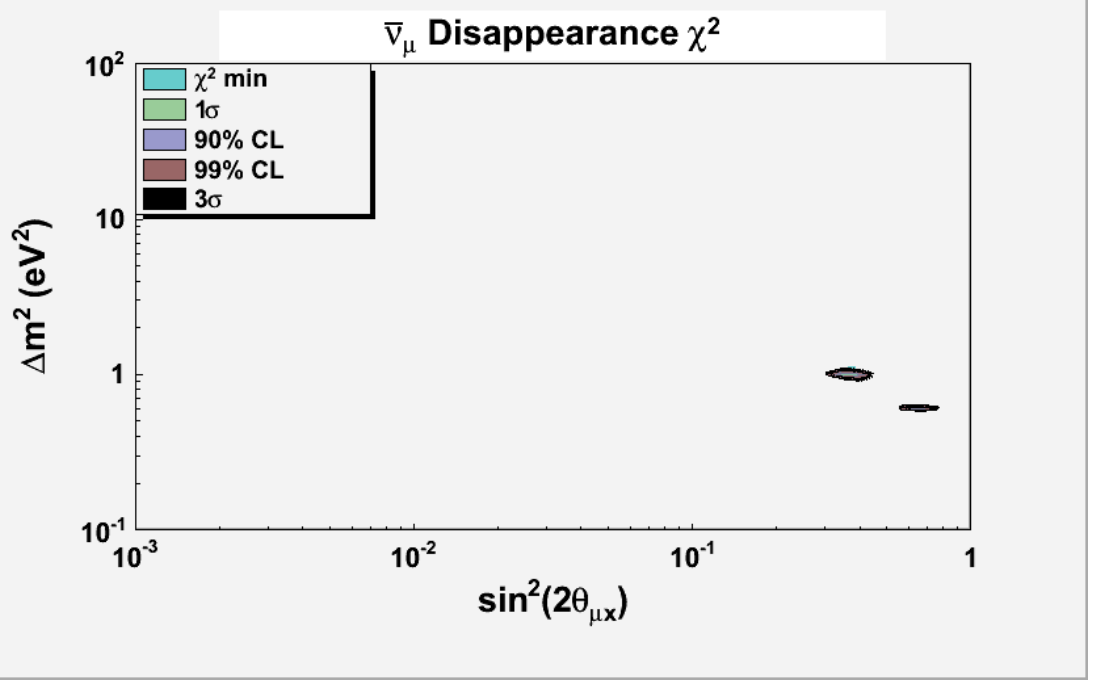

Figure 38: The BooNE allowed region for $\bar{\nu}_{\mu}$ disappearance at the global antineutrino best-fit point $\Delta m_{14}^{2}=0.915 \mathrm{eV}^{2}$ and $\sin ^{2} 2 \theta_{\mu \mu}=0.35$. 
$\sin ^{2} 2 \theta_{\mu \mu} \sim 3 \%$ for neutrinos and $\sin ^{2} 2 \theta_{\mu \mu} \sim 5 \%$ for antineutrinos.

\section{Other Experiments}

The MINOS experiment has measured neutrino oscillations at the atmospheric scale with $\nu_{\mu}$ disappearance 24 and has begun to look at $\bar{\nu}_{\mu}$ disappearance ${ }^{34}$ ). It is interesting to note that the MINOS antineutrino data so far are consistent with the antineutrino $3+1$ model of reference ${ }^{12}$ and consistent with $\bar{\nu}_{\mu}$ disappearance at the LSND scale. Fig. 39 shows the MINOS $\bar{\nu}_{\mu}$ event rate as a function of reconstructed neutrino energy compared to the Monte Carlo expectation. Although the statistics are low, the MINOS $\bar{\nu}_{\mu}$ event rate is suppressed above $10 \mathrm{GeV}$, where there should be almost no suppression due to atmospheric neutrino oscillations. Fig. 40 shows the MINOS $\bar{\nu}_{\mu}$ disappearance allowed region, which is consistent with oscillations at the $\sim 1 \mathrm{eV}^{2}$ scale.

The only other experiment or proposal that is capable of addressing these physics objectives at the $\sim 1 \mathrm{eV}^{2}$ scale is a Letter of Intent to refurbish the CERN PS neutrino beam and build two liquid argon detectors 35). However, the proposed BooNE experiment, with the existing BNB, should be able to obtain results prior to the CERN experiment.

\section{Cost Estimate}

Table 9 shows a breakdown of the cost estimate for constructing a second BooNE detector in a near location. The estimate is based on the MiniBooNE construction costs. The total estimated cost is $\$ 7.3 \mathrm{M}$, including contingency $(\sim 30 \%)$ and escalation (3\% per year). The BooNE construction is assumed to start in 2010 and last for 3 years. The estimated cost for moving MiniBooNE to a near location is $\sim \$ 4 \mathrm{M}$. An additional advantage of moving MiniBooNE is that the MicroBooNE detector could then be moved into the original MiniBooNE enclosure, thereby saving the expense of building a new MicroBooNE enclosure.

\section{References}

1) A. Aguilar-Arevalo et al., Phys. Rev. Lett. 98, 231801 (2007); A. Aguilar-Arevalo et al., Phys. Rev. Lett. 102, 101802 (2009).

2) Jeffrey A. Harvey, Christopher T. Hill, Richard J. Hill, arXiv:0708.1281; arXiv:0712.1230.

3) S. N. Gninenko, [arXiv:00902.3802]; S. N. Gninenko and D. S. Gorbunov, [arXiv:00907.4666].

4) Michel Sorel, Janet Conrad, and Michael Shaevitz, Phys. Rev. D 70, 073004 (2004); G. Karagiorgi et al., Phys. Rev. D 75, 013011 (2007); Alessandro Melchiorri et al., arXiv:0810.5133.

5) Heinrich Paes, Sandip Pakvasa, Thomas J. Weiler, Phys. Rev. D 72, 095017 (2005); arXiv:hep-ph/0504096.

6) T. Goldman, G. J. Stephenson Jr., and B. H. J. McKellar, Phys. Rev. D 75, 091301 (2007).

7) Michele Maltoni and Thomas Schwetz, Phys. Rev. D 76, 093005 (2007); arXiv:0705.0197.

8) Ann E. Nelson and Jonathan Walsh, arXiv:0711.1363. 


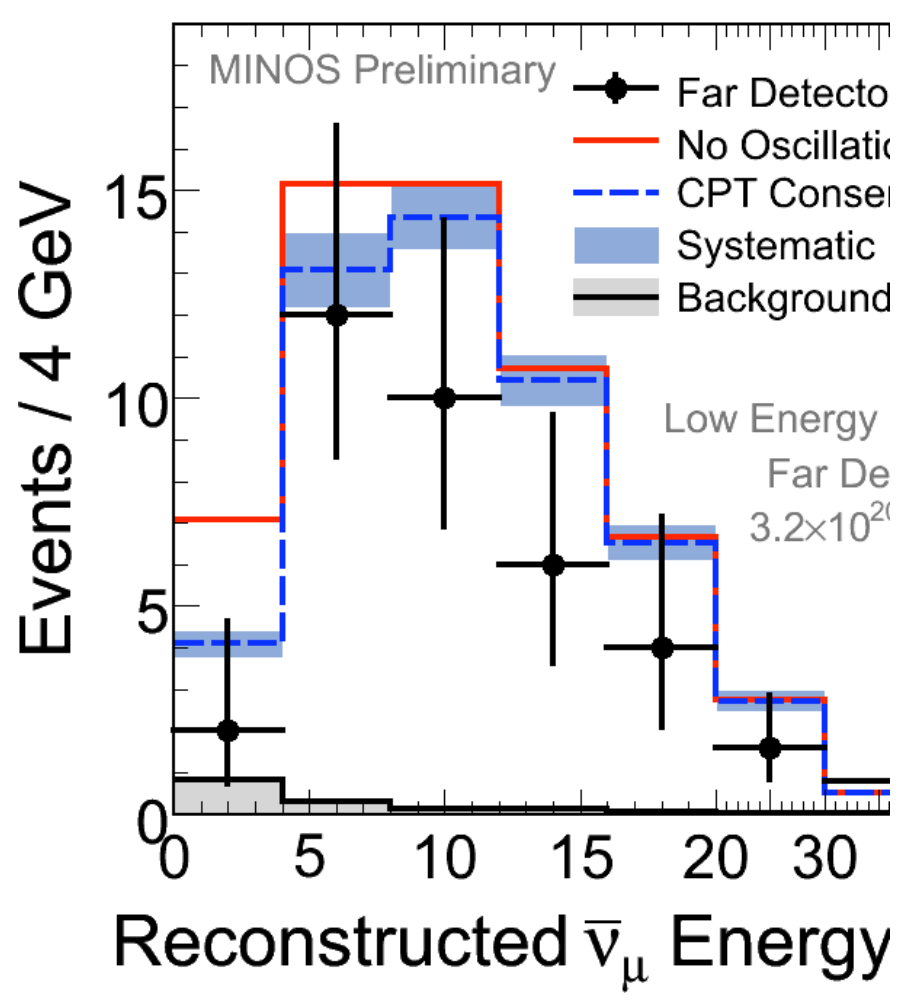

Figure 39: The MINOS $\bar{\nu}_{\mu}$ event rate as a function of reconstructed neutrino energy compared to the Monte Carlo expectation. Although the statistics are low, the MINOS $\bar{\nu}_{\mu}$ event rate is suppressed above $10 \mathrm{GeV}$, where there should be almost no suppression due to atmospheric neutrino oscillations.

\begin{tabular}{|c|c|}
\hline Item & Cost $(\$ \mathrm{~K})$ \\
\hline \hline Tank and support structure & 1065 \\
\hline PMT's & 1759 \\
\hline Electronics/DAQ & 512 \\
\hline Oil & 1429 \\
\hline Calibrations & 412 \\
\hline Miscellaneous & 198 \\
\hline Engineering \& Construction & 1894 \\
\hline \hline Total & 7269 \\
\hline
\end{tabular}

Table 9: A breakdown of the cost estimate for constructing a second BooNE detector in a near location, including contingency and escalation. 


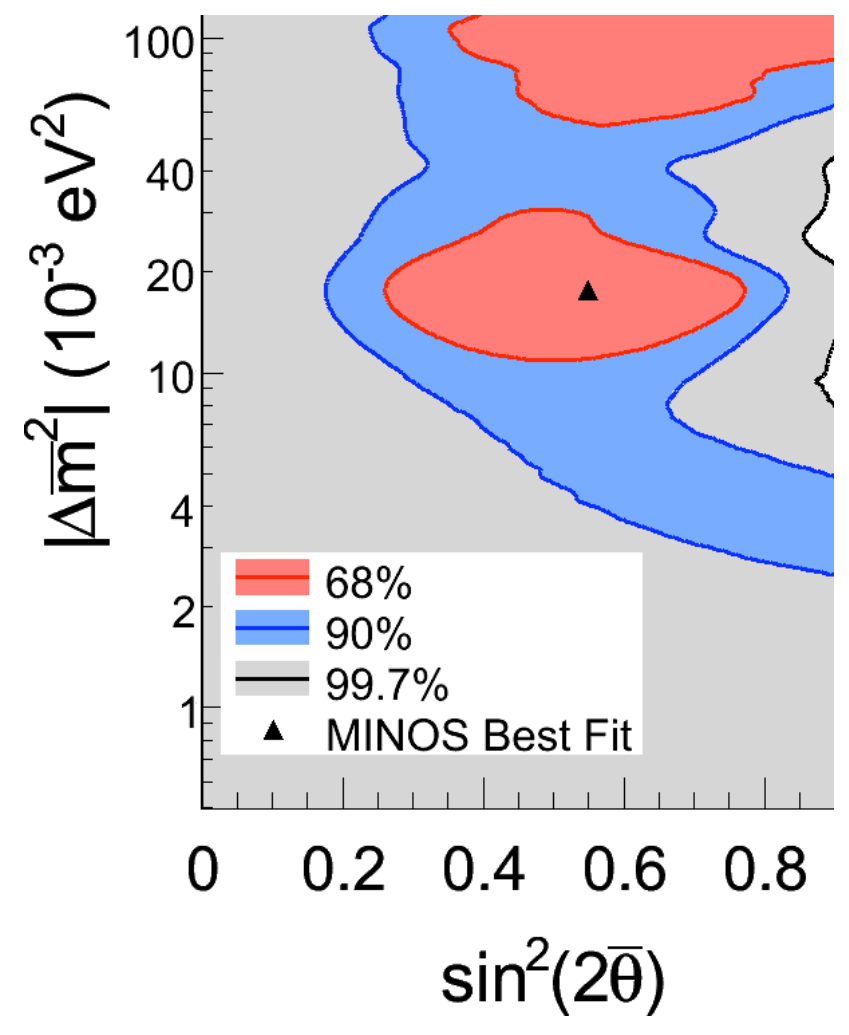

Figure 40: The MINOS $\bar{\nu}_{\mu}$ disappearance allowed region, which is consistent with oscillations at the $\sim 1 e V^{2}$ scale. 
9) Sebastian Hollenberg and Heinrich Paes, [arXiv:00904.2167].

10) C. Athanassopoulos et al., Phys. Rev. Lett. 75, 2650 (1995); 77, 3082 (1996); 81, 1774 (1998); A. Aguilar et al., Phys. Rev. D 64, 112007 (2001).

11) A. Aguilar-Arevalo et al., Phys. Rev. Lett. 103, 111801 (2009).

12) G. Karagiorgi et al., arXiv:0906.1997.

13) B. T. Cleveland et al., Astrophys. J. 496, 505 (1998).

14) J. N. Abdurashitov et al., Phys. Rev. C 60, 055801 (1999).

15) W. Hampel et al., Phys. Lett. B 447, 127 (1999).

16) S. Fukuda et al., Phys. Lett. B 539, 179 (2002).

17) Q. R. Ahmad et al., Phys. Rev. Lett. 87, 071301 (2001); Q. R. Ahmad et al., Phys. Rev. Lett. 89, 011301 (2002); S. N. Ahmed et al., Phys. Rev. Lett. 92, 181301 (2004).

18) K. Eguchi et al., Phys. Rev. Lett. 90, 021802 (2003); T. Araki et al., Phys. Rev. Lett. 94, $081801(2005)$.

19) K. S. Hirata et al., Phys. Lett. B 280, 146 (1992); Y. Fukuda et al., Phys. Lett. B 335, 237 (1994).

20) Y. Fukuda et al., Phys. Rev. Lett. 81, 1562 (1998).

21) W. W. M. Allison et al., Phys. Lett. B 449, 137 (1999).

22) M. Ambrosio et al., Phys. Lett. B 517, 59 (2001).

23) M. H. Ahn et al., Phys. Rev. Lett. 90, 041801 (2003).

24) D. G. Michael et al., Phys. Rev. Lett. 97, 191801 (2006); P. Adamson et al., Phys. Rev. Lett. 101, 131802 (2008).

25) T. Katori, A. Kostelecky and R. Tayloe, Phys. Rev. D 74, 105009 (2006).

26) G. Barenboim and J. D. Lykken, Phys. Lett. B 554, 73 (2003); arXiv:0908.2993.

27) A. Aguilar-Arevalo et al., Phys. Rev. D 79, 072002 (2009).

28) A. Aguilar-Arevalo et al., Nucl. Instr. Meth. A599, 28 (2009).

29) A. Aguilar-Arevalo et al., Phys. Rev. Lett. 100, 032301 (2008).

30) A. Aguilar-Arevalo et al., Phys. Lett. 664B, 41 (2008).

31) P. Adamson et al., Phys. Rev. Lett. 102, 211801 (2009).

32) A. Aguilar-Arevalo et al., Phys. Rev. Lett. 103, 061802 (2009).

33) M. G. Catanesi et al., Eur. Phys. J. C 52, 29 (2007) arXiv:hep-ex/0702024.

34) MINOS, J. Hartnell (2009), FNAL Seminar, 15 May $2009 . \quad$ URL: http://theory.fnal.gov/jetp/talkes/Hartnell.pdf.

35) B. Baibussinov et al., arXiv:0009.0355. 